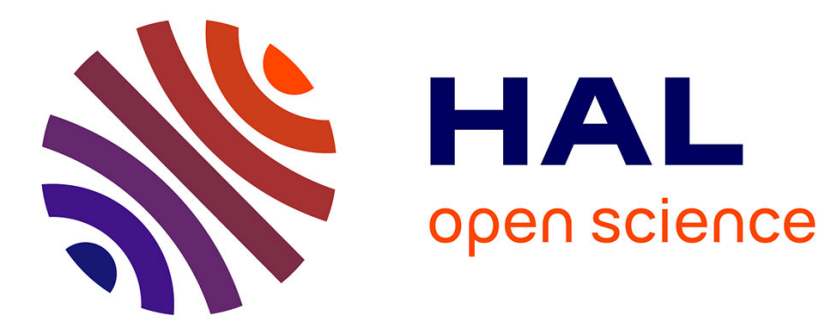

\title{
Athermal dislocation strengthening in UO2
}

Luc Portelette, Jonathan Amodeo, Ronan Madec, Bruno Michel

\section{To cite this version:}

Luc Portelette, Jonathan Amodeo, Ronan Madec, Bruno Michel. Athermal dislocation strengthening in UO2. Journal of Nuclear Materials, 2020. hal-02938715

\section{HAL Id: hal-02938715 https://hal.science/hal-02938715}

Submitted on 15 Sep 2020

HAL is a multi-disciplinary open access archive for the deposit and dissemination of scientific research documents, whether they are published or not. The documents may come from teaching and research institutions in France or abroad, or from public or private research centers.
L'archive ouverte pluridisciplinaire HAL, est destinée au dépôt et à la diffusion de documents scientifiques de niveau recherche, publiés ou non, émanant des établissements d'enseignement et de recherche français ou étrangers, des laboratoires publics ou privés. 


\section{Athermal dislocation strengthening in $\mathrm{UO}_{2}$}

Luc Portelette ${ }^{1}$, Jonathan Amodeo ${ }^{2}$, Bruno Michel ${ }^{1 *}$, Ronan Madec ${ }^{3}$

${ }^{1}$ CEA, DEN, DEC, SESC, LSC bat 151 Centre de Cadarache F-13108 Saint Paul Lez Durance, France

2 Université de Lyon, INSA-Lyon, CNRS, MATEIS UMR5510, F-69621 Villeurbanne, France

3 CEA, DAM, DIF, F-91297 Arpajon, France

Published in Journal of Nuclear Materials as:

L. Portelette, J. Amodeo, B. Michel, R. Madec, " Athermal dislocation strengthening in UO2", Journal of Nuclear Materials, 538 (2020) 152157

$\left(^{*}\right)$ Corresponding author:

Bruno Michel

bruno.michel@cea.fr 


\title{
Athermal dislocation strengthening in $\mathrm{UO}_{2}$
}

\begin{abstract}
Understanding the mechanical behavior of uranium dioxide (UO2) at hightemperature is of great interest to predict the behavior of the nuclear fuel under incidental operating conditions. Here, strengthening elementary processes and dislocation hardening coefficients are studied by the mean of Dislocation Dynamics (DD) simulations in $\mathrm{UO}_{2}$. The three slip modes of the fluorite structure i.e., $1 / 2<110>\{100\}, 1 / 2<110>\{110\}$ and $1 / 2<110>\{111\}$ are modelled and all the possible dislocation configurations are analyzed and discussed. Averaged hardening coefficients are derived and their sensitivity to the sample orientation is discussed in the light of the seminal experimental work of Sawbridge and Sykes. This DD study gives rise to new insights about $\mathrm{UO}_{2}$ mechanical behavior at high-temperature.
\end{abstract}

\section{Introduction}

Uranium dioxide $\left(\mathrm{UO}_{2}\right)$ is the main primary fuel material of nuclear power plant. The understanding of its mechanical behavior is of first glance, especially in the context of incidental operating conditions of the reactor, where the cladding tube can have a mechanical interaction with the pellet. This pellet-to-cladding reaction has to be considered regarding its potential impact on the structural integrity of the cladding, which is the first confinement barrier for radioactive elements. While the macroscopic link between the fuel pellet mechanical properties and the cladding tube were qualitatively drawn [1-3], several multiphysics aspects including mechanical, thermal and irradiation considerations are still required to better understand the multi-scale context of the fuel evolution. In this study, we will focus on the deformation and strain-hardening of nonirradiated $\mathrm{UO}_{2}$ single crystals at high-temperature $(T>1350 \mathrm{~K})$, in a temperature regime relative to incidental conditions. 
$\mathrm{UO}_{2}$ has the fluorite structure $\left(a_{0}=5.47 \AA\right)$ and is characterized by a brittle-to-ductile transition for temperatures larger than $800 \mathrm{~K}$. At these temperatures, dislocation glide occurs in $1 / 2<110>\{100\}$ and $1 / 2<110>\{110\}$ slip systems (referred respectively as modes I and II in the following) and, more controversially, in $1 / 2<110>\{111\}$ (mode III) [4-13]. Single crystal Critical Resolved Shear Stresses (CRSS) in $\{100\}$ and $\{110\}$ show significant variations when varying the temperature, typical of materials with high lattice friction. These variations are attributed to thermally-activated dislocation glide processes (e.g., kink-pair mechanism) $[10,11,14,15]$. Very few is known about the athermal temperature transition $T_{a}$ (the temperature at which CRSS reach a plateau) in $\mathrm{UO}_{2}$. In the original work of Lefebvre et al. [10], $T_{a}^{I}$ is expected to be close to $1400 \mathrm{~K}$ whereas Portelette et al. [16] recently claim a larger value of $T_{a}^{I}=1750 \mathrm{~K}$ by combining CRSS experimental measurements from different sources. Given the same temperature range, dislocation slip in $1 / 2<110>\{110\}$ is characterized by slightly larger CRSS values that continuously decrease rising the temperature up to $T_{a}^{I I} \sim 2000 \mathrm{~K}$ [16]. Nonetheless, one can assume that mode II is characterized by a weak lattice friction for $T>1350 \mathrm{~K}$ and CRSS of few tens of MPa only. Dislocation glide has not been yet isolated within mode III slip systems and no CRSS (and no $T_{a}^{I I I}$ ) were derived up to now. Indeed, very few TEM studies dedicated to dislocations in $\mathrm{UO}_{2}$ exist in the literature [7,9-11].

Atomistic simulations using semi-empirical potentials were performed in $\mathrm{UO}_{2}$ to investigate CRSS versus temperature variations [15,17,18]. Fossati et al. [17] show that $1 / 2<110>\{100\}$ is the softer slip mode using molecular dynamics (MD). On the other hand, the authors show that $1 / 2<110>\{110\}$ is harder than $1 / 2<110>\{111\}$. Lunev et al. used MD to investigate more in the details the elementary processes responsible for the mobility of $1 / 2<110>\{100\}$ edge [14] and $1 / 2<110>\{110\}$ screw [19] dislocations. In addition, Soulié [15] used the variable charges SMTBQ parameterization and molecular statics to describe $\{100\}$ edge dislocation gliding by a kink-pair process. While these atomistic studies provide some information on isolated dislocations (kink-pairs, defect clusters formation, self-pinning), none of them can give a clear description of $\mathrm{UO}_{2}$ single crystal deformation.

In the temperature range relative to incidental conditions $(T>1350 \mathrm{~K}), 1 / 2<110>\{100\}$ CRSS in $\mathrm{UO}_{2}$ are particularly close to the athermal transition $[7,10,11,15-17]$. At $\mathrm{T}=1600$ $\mathrm{K}$, Portelette and coauthors emphasized that a complex slip activity improves partially the 
description of the plastic anisotropy observed in the experiments presuming of the important role of the forest hardening (typical of the athermal regime). In the athermal regime, forest hardening is usually rationalized by the mean of the generalized Taylor's equation (equation (1)) and dislocation interaction coefficients ( $a^{s u}$ ) as introduced by Franciosi [20].

$$
\tau_{c}^{s}=\mu b \sqrt{\sum_{u} a^{s u} \rho^{u}}
$$

Where $\tau_{c}^{s}$ is the critical shear stress for dislocation slip in system $s$ crossing $u$ forest systems with $\rho^{u}$ the dislocation density in each forest system. $\mu$ is the shear modulus and $b$ is the magnitude of the Burgers. $a^{s u}$ is the dislocation interaction coefficient between the systems $s$ and $u$. This equation is derived from Taylor's equation [21,22] where a mean interaction coefficient $\alpha$ is used.

In this study, we propose to use Dislocation Dynamics (DD) to investigate the strengthening of $\mathrm{UO}_{2}$ single crystal within the athermal regime to better capture $\mathrm{UO}_{2}$ mechanical behavior under incidental conditions. DD is a mesoscale simulation method originally developed to study the collective behavior of dislocations [23-25]. When used with periodic boundary conditions [26,27], DD simulations are particularly useful to study the bulk material mechanical response using a representative volume element. In particular, it allows to correlate the stress response of the crystal relatively to its dislocation density content. Recently, many studies took the advantage of DD simulations to determine dislocation interaction coefficients using equation (1) as e.g., in FCC crystals [28-32], in BCC crystals [32-34], in HCP crystals [31,35] and other complex crystallography such as MgO [36] and olivine [37].

Here, $\mathrm{DD}$ is applied to $\mathrm{UO}_{2}$ fluorite where first, dislocation-dislocation interactions are investigated computing dislocation reaction maps and using several elastic models. Thus, dislocation interaction coefficients are estimated and compared to values obtained for several other materials. Thus, averaged coefficients are derived using the composition law (equation (1)) and the influence of orientations is addressed. Finally, results are discussed 
leading to an interpretation of the seminal mechanical experiments of Sawbridge and Sykes in $\mathrm{UO}_{2}$ single crystal [8].

\section{Slip systems, interactions and symmetries in $\mathrm{UO}_{2}$}

\section{1 $\mathrm{UO}_{2}$ slip systems}

$\mathrm{UO}_{2}$ deformation is characterized by dislocation glide in $1 / 2<110>\{100\}, 1 / 2<110>\{110\}$ and , possibly, $1 / 2<110>\{111\}$ slip systems. Table 1 references the slip systems of the fluorite structure. Burgers vectors are numbered from 1 to 6 and letters are used to characterize individual slip plane i.e., from $A$ to $C$ for the $\{100\}$ planes, from $A$ to $F$ for the six $\{110\}$ slip systems and from A to D for $\{111\}$ slip, following the Schmid and Boas notation [38]. 
Table 1: List of $\mathrm{UO}_{2}$ slip systems. Burgers vectors and the $\{111\}$ slip mode are labeled using the Schmid and Boas notation [38]. Additional notations are proposed for $\{100\}$ and $\{110\}$ slip modes and a list of slip systems is provided for each Burges vectors.

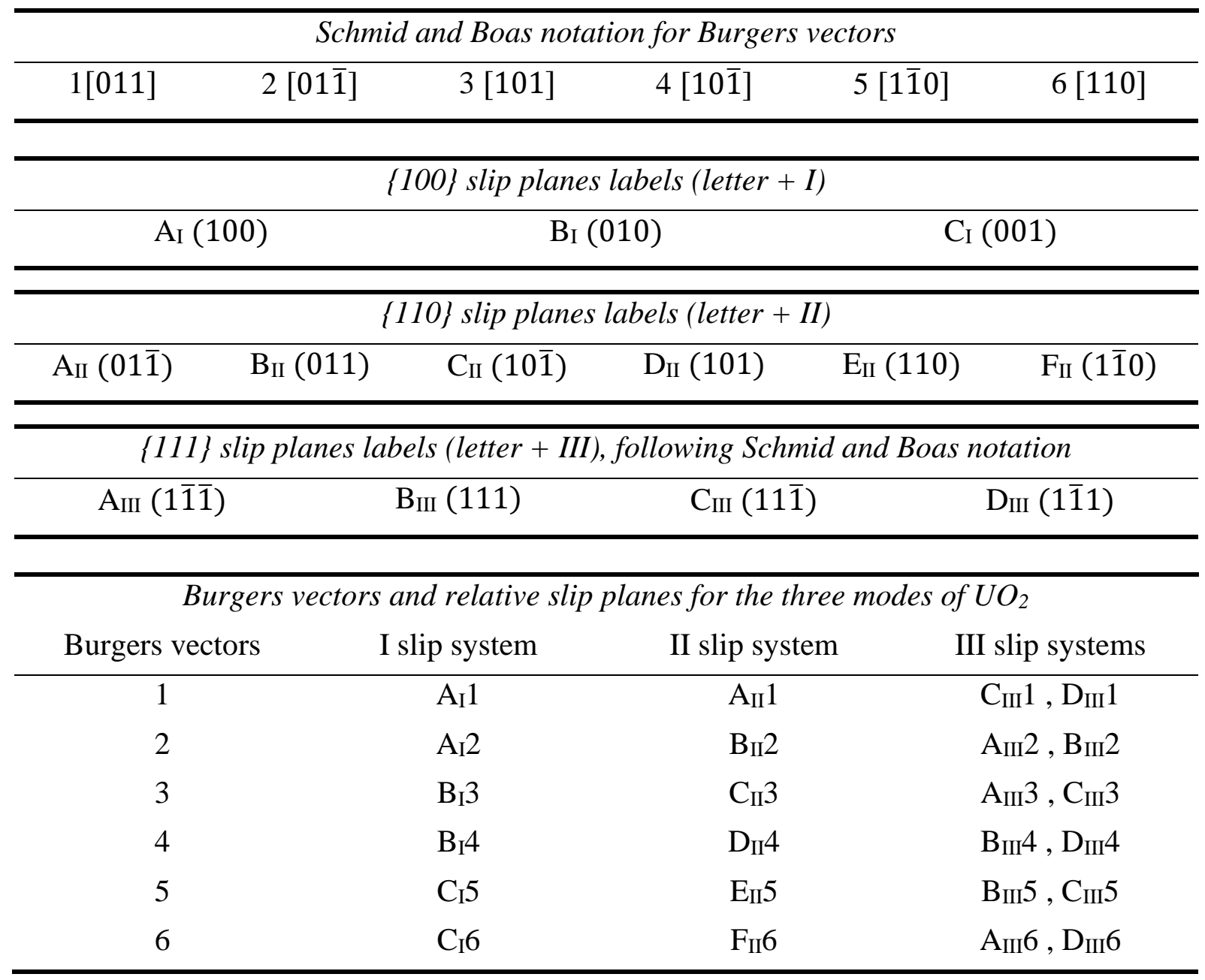

\subsection{Symmetries and dislocation reactions in $\mathrm{UO}_{2}$}

Table 2: Interactions between dislocations in $\mathrm{UO}_{2}$. " $\alpha_{i}$ " refers to the coefficient index of the strengthening matrix (see Table S1 in supplementary materials). "Example of slip system interaction" provides a typical pair of slip systems using Table 1 denomination (primary and forest systems are ranked respectively first and second, except for stared coefficients). "Interaction ID" are labelled as self, dipole, glissile, Hirth, collinear and sessile. Self and dipole account respectively for self (without distinction between slip modes) and dipolar interactions when interacting Burgers vectors differ. Glissile, Hirth, collinear and sessile refer to the different types of junctions. Dipoles and junctions are labeled using intersecting modes, as subscript, and specific property, as junction plane or reaction orientation, as superscript. Glissile refers to junctions lying in fluorite crystallographic slip plane. Note that the FCC Lomer junction which is originally sessile in metals is glissile in $\mathrm{UO}_{2}$, and referenced as glissile $e_{I I I / I I I}^{I, L}$. Sessile is for a/2<110> junction not lying in fluorite crystallographic slip plane. Hirth refers for weaker 
junctions with a a<100> Burgers vectors. Collinear is for collinear interaction and is labeled using the angle between cross slip planes, as superscript.

\begin{tabular}{|c|c|c|c|c|c|c|c|}
\hline & $\alpha_{i}$ & $\begin{array}{l}\text { Example of } \\
\text { slip system } \\
\text { interaction }\end{array}$ & $\begin{array}{l}\text { Interaction } \\
\text { ID }\end{array}$ & & $\alpha_{i}$ & $\begin{array}{l}\text { Example of } \\
\text { slip system } \\
\text { interaction }\end{array}$ & $\begin{array}{c}\text { Interaction } \\
\text { ID }\end{array}$ \\
\hline \multirow{3}{*}{$I / I$} & $\alpha_{0}^{\prime}$ & $\mathrm{A}_{\mathrm{I}} 1, \mathrm{~A}_{\mathrm{I}} 1$ & self & \multirow{2}{*}{$I I I / I I I$} & $\alpha_{11}$ & $\mathrm{C}_{\mathrm{III}} 1, \mathrm{C}_{\mathrm{III}} 3$ & dipole $_{I I I / I I I}$ \\
\hline & $\alpha_{1}$ & $\mathrm{~A}_{\mathrm{I}} 1, \mathrm{~A}_{\mathrm{I}} 2$ & dipole $_{I / I}$ & & $\alpha_{12}$ & $\mathrm{C}_{\mathrm{III}} 1, \mathrm{~B}_{\mathrm{III}} 4$ & glissile $_{I I I / I I I}^{I, L}$ \\
\hline & $\alpha_{2}$ & $\mathrm{~A}_{\mathrm{I}} 1, \mathrm{~B}_{\mathrm{I}} 3$ & glissile ${ }_{I / I}^{I I}$ & \multirow{4}{*}{$I / I I I$} & $\alpha_{13}, \alpha_{13} *$ & $\mathrm{~A}_{\mathrm{I}} 1, \mathrm{C}_{\mathrm{III}} 1$ & collinear $r_{I / I I I}^{55^{\circ}}$ \\
\hline \multirow[b]{2}{*}{$I I / I I$} & $\alpha_{3}$ & $\mathrm{~A}_{\mathrm{III}} 1, \mathrm{~B}_{\mathrm{II}} 2$ & Hirth $_{I I / I I}$ & & $\alpha_{14}, \alpha_{14} *$ & $\mathrm{~A}_{\mathrm{I}} 1, \mathrm{~A}_{\mathrm{III}} 2$ & Hirth $_{I / I I I}$ \\
\hline & $\alpha_{4}$ & $\mathrm{~A}_{\text {II }} 1, \mathrm{C}_{\text {II }} 3$ & sessile $_{I I / I I}^{\{112\}}$ & & $\alpha_{15}, \alpha_{15} *$ & $\mathrm{~A}_{\mathrm{I}} 1, \mathrm{~A}_{\text {III }} 3$ & glissile $_{I / I I I a}^{I I I}$ \\
\hline \multirow{3}{*}{$I / I I$} & $\alpha_{5}, \alpha_{5}^{*}$ & $\mathrm{~A}_{\mathrm{I}} 1, \mathrm{~A}_{\mathrm{II}} 1$ & collinear $r_{I / I I}^{90^{\circ}}$ & & $\alpha_{16}, \alpha_{16} *$ & $\mathrm{~A}_{\mathrm{I}} 1, \mathrm{C}_{\mathrm{III}} 3$ & glissile $_{I / I I I b}^{I I I}$ \\
\hline & $\alpha_{6}, \alpha_{6} *$ & $\mathrm{~A}_{\mathrm{I}} 1, \mathrm{~B}_{\mathrm{II}} 2$ & $\operatorname{Hirth}_{I / I I}$ & \multirow{5}{*}{$I I / I I I$} & $\alpha_{17}, \alpha_{17} *$ & $\mathrm{~A}_{\mathrm{II}} 1, \mathrm{C}_{\mathrm{III}} 1$ & collinear $_{I I / I I}^{35^{\circ}}$ \\
\hline & $\alpha_{7}, \alpha_{7} *$ & $\mathrm{~A}_{\mathrm{I}} 1, \mathrm{C}_{\mathrm{II}} 3$ & glissile $_{I / I I}^{I}$ & & $\alpha_{18}, \alpha_{18} *$ & $\mathrm{~A}_{\mathrm{II}} 1, \mathrm{~A}_{\mathrm{III}} 2$ & $\operatorname{Hirth}_{I I / I I I}^{\{012\}}$ \\
\hline \multirow{3}{*}{$I I I / I I I$} & $\alpha_{8}$ & $\mathrm{C}_{\mathrm{III}} 1, \mathrm{D}_{\mathrm{III}} 1$ & collinear $r_{I I I / I I}^{70^{\circ}}$ & & $\alpha_{19}, \alpha_{19} *$ & $\mathrm{~A}_{\mathrm{II}} 1, \mathrm{~A}_{\mathrm{III}} 3$ & $\operatorname{sessile}_{I I / I I I}^{\{113\}}$ \\
\hline & $\alpha_{9}$ & $\mathrm{C}_{\mathrm{III}} 1, \mathrm{~A}_{\mathrm{III}} 2$ & $\operatorname{Hirth}_{I I I / I I I}$ & & $\alpha_{20}, \alpha_{20} *$ & $\mathrm{~A}_{\mathrm{II}} 1, \mathrm{C}_{\mathrm{III}} 3$ & glissile $_{I I / I I I}^{I I I}$ \\
\hline & $\alpha_{10}, \alpha_{10} *$ & $\mathrm{C}_{\text {IIII }} 1, \mathrm{~A}_{\mathrm{III}} 3$ & glissile $_{I I I / I I I}^{I I I}$ & & & & \\
\hline
\end{tabular}

Due to the particularly high number of slip systems in $\mathrm{UO}_{2}, 576$ slip systems interactions can be defined. With the help of crystallographic symmetries, this amount is reduced to 33 as shown by the strengthening matrix (see Table S1 in the supplementary). The notation $\alpha_{i}=\sqrt{a_{i}}$ is used here to write down strengthening matrix coefficients $\alpha^{s u}$. Each $\alpha_{i}$ refers to a unique strengthening coefficient and, thus, to a particular dislocationdislocation interaction. Stared coefficients $\alpha_{i}^{*}$ are defined for asymmetric reactions i.e., when two different strengthening coefficients are derived when switching primary and forest slip systems in the simulation (see [32] for more details).

In the following, all possible interactions between slip systems in $\mathrm{UO}_{2}$ are investigated i.e., within $\{100\}$ and $\{110\}$ slip modes (referred as I/I, II/II and I/II), these interactions are the most probable to appear in $\mathrm{UO}_{2}$ single crystal experiments. But also, among $\{111\}$ slip systems (called III/III) that are similar to FCC metal dislocation interactions as well as crossed interactions between $\{111\}$ and the two dominant slip modes (later referred as I/III and II/III). Table 2 summarizes slip system interactions and induced reaction products (labelled as Interaction ID) referred as $X_{a / b}^{c}$, where $X$ refers to the reaction name, $a$ and $b$ to the involved modes, and $c$ to a specific information about the reaction (e.g., the glide plane of a junction or the angle between the slip planes in the case of collinear 
reactions). Please note that in the case of multiple reaction products (e.g., junctions in addition to crossed states and repulsions), the interaction name will only refer to the strongest reaction.

Modes I and II are characterized by few contact reactions only. $\alpha_{2}$ refers to the glissile $_{I / I}^{I I}$ junction, which is induced by I/I interactions but gliding in mode II slip systems. On the other hand, $\alpha_{3}$ and $\alpha_{4}$ deduced from II/II interactions lead to two sessile junctions, the $\operatorname{Hirth}_{I I / I I}$ and the sessile $\{11 / I I\}$, the latter being supposed to glide in $\{112\}$ which is not a slip plane of the fluorite structure. The self-interaction is labelled $\alpha_{0}$ ' due to the coupling between dipolar and collinear interactions (see Devincre et al. [39]) and $\alpha_{1}$ relies on the dipolar interaction dipole $_{I / I}$ within mode I slip systems. I/II crossed configurations lead to asymmetrical coefficients labelled $\alpha_{5}, \alpha_{6}$ and $\alpha_{7}$. All I/I, II/II and I/II contact reactions were originally described for MgO by Carrez et al. [40] and Amodeo et al. [36], where reaction maps were computed using the isotropic assumption. In addition, $\alpha_{i}$ with $13 \leq i \leq 16$ and $17 \leq i \leq 20$ respectively rely on I/III and II/III crossed interactions. These last coefficients are also asymmetrical. Several examples of I/III and II/III reaction maps are described in the following. III/III interactions (here referenced by $\alpha_{i}$ with $8 \leq i \leq 12$ ) were widely investigated in the case of FCC metals (see e.g., Madec et al. [41]). In the next section, forest coefficients for $\mathrm{UO}_{2}$ are discussed for all $\alpha_{i}$ configurations presented in Table 2

\section{Simulation methods}

\subsection{Dislocation interaction mapping}

DD simulations are performed using the MobiDiC code [32,42]. As the basics of latticebased DD codes are already widely detailed in the literature (see for example [24]) here we only focus on the specific aspects of the following simulations.

To study dislocation contact reactions, two initially straight dislocations of same length $(2 \mu \mathrm{m})$ that intersect at their mid-point, are relaxed elastically using DD simulations. As shown Figure 1, parent dislocations are characterized by the two angles $\beta_{1}$ and $\beta_{2}$ while $\beta_{j}$ is the angle between the recombination direction and the associated dislocation Burgers vectors. As product results depend on the relative orientation of parent dislocations (later 
referred as $\phi_{1}$ and $\phi_{2}$ ), several types of reaction are observed: (i) the repulsion of the two dislocations, (ii) a crossed state, when attractive dislocations just stay in contact without merging, (iii) a recombination of the two parent dislocations forming a junction (or a collinear reaction leading to annihilation), when it induces a global line energy gain. The characteristics of a junction depend of the parent dislocations. The Burgers vector of the junction is defined by $\overrightarrow{b_{J}}=\overrightarrow{b_{1}}+\overrightarrow{b_{2}}$ where indices 1 and 2 rely on the two parent dislocations, its line vector is $\overrightarrow{l_{j}}=\overrightarrow{n_{1}} \times \overrightarrow{n_{2}}$ and thus, the slip plane $\overrightarrow{n_{j}}$ of the junction is derived using the two aforementioned quantities $\overrightarrow{n_{\jmath}}=\overrightarrow{b_{\jmath}} \times \vec{l}_{\jmath}$. A junction is asymmetrical when $\beta_{1} \neq \beta_{2}$ or when Poisson's ratios between slip systems strongly differ as discussed later.

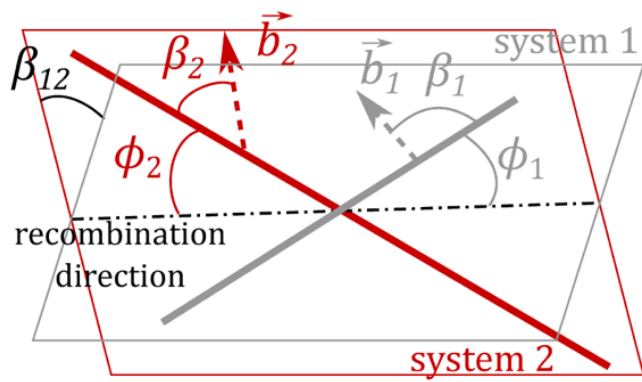

(a)

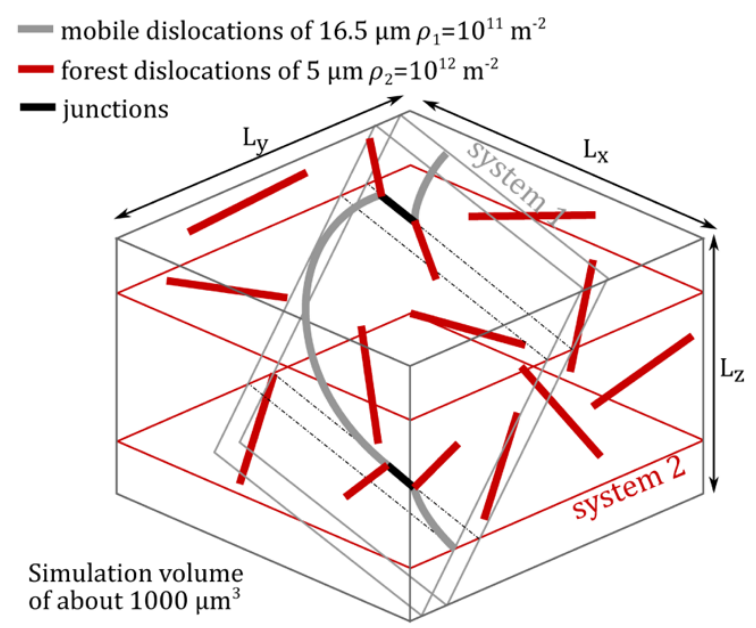

Figure 1: (a) Dislocation configuration for reaction maps computation, (b) Schematic representation of the simulation setup for dislocation interaction coefficient (only some of the dislocations are sketched).

In this study, dislocation reaction maps are computed using both the analytical approach based on the straight dislocation approximation proposed by Püschl [43] and DD simulations where the segments discretization allows lines curvature. In addition, boundaries between crossed and repulsive states are also provided using the isotropic elasticity theory in the framework of infinite dislocations [44]. In the results section, anisotropic and Bacon-Scattergood (BS) approximations of straight dislocations are drawn together. 
Dislocation reaction maps weakly depend on temperature (that is accounted only in the elastic constants). Indeed, these are minimization simulations without external loading. They are thus designed for the description of dislocation interactions in athermal (for $T>T_{a}$ ) and quasi-static conditions, where the influence of the dislocation velocity on the plastic response is believed to be negligible. Line energies used for reaction maps simulations are computed using DisDi $[45,46]$ and anisotropic elastic constants at $T=1373$ $\mathrm{K}$ [47]. Indeed, this temperature reflects the most anisotropic conditions in the temperature range of the fuel incidental transient i.e., for $T$ ranging from $1350 \mathrm{~K}$ to 2000 K. While dislocation-dislocation interaction coefficient will be computed at larger temperature, $T=1373 \mathrm{~K}$ allows to better quantify the spread of dislocation-dislocation interactions induced by the chosen elastic theory, without downgrading the main results. Anisotropic energies of screw and edge dislocations are used to obtain BS Poisson's ratio $v^{B S}$ and shear modulus $\mu^{B S}$ for each slip mode [32]. BS elastic constants are provided Table 3. More details on $\mathrm{UO}_{2}$ elastic properties are provided in the section $\mathrm{S} 2$ of the supplementary.

Table 3: BS elastic constants for the three slip modes at $1373 \mathrm{~K}$ and $1973 \mathrm{~K}$.

\begin{tabular}{llccc}
\hline & & $1 / 2<110>\{100\}$ & $1 / 2<110>\{110\}$ & $1 / 2<110>\{111\}$ \\
\hline $1373 \mathrm{~K}$ & $v^{B S}$ & 0.25 & 0.32 & 0.29 \\
& $\mu^{B S}(\mathrm{GPa})$ & & 72.7 & \\
$1973 \mathrm{~K}$ & $v^{B S}$ & 0.27 & 0.32 & 0.30 \\
& $\mu^{B S}(\mathrm{GPa})$ & & 61.4 & \\
\hline
\end{tabular}

\subsection{DD simulation of interaction coefficients}

Dislocation-dislocation interaction coefficients are computed using the standard method described e.g., in refs. [28,32]. Here, an orthorhombic simulation cell of approximatively $1000 \mu \mathrm{m}^{3}$ is considered and simulations are performed using periodic boundary conditions [27]. Forest dislocations of $5 \mu \mathrm{m}$ length are randomly distributed in the cell within a given system $u$. All dislocation characters are equivalently represented and the forest dislocation density $\rho^{u}$ is of about $10^{12} \mathrm{~m}^{-2}$ (the procedure to deduce the coefficient value at a different dislocation density is provided in [32]). The dislocation density of the mobile system $s$ is of $10^{11} \mathrm{~m}^{-2}$ with dislocations of $16.5 \mu \mathrm{m}$ length. Accounting for crystal symmetries, only few slip systems are used to investigate all 
strengthening coefficients. More details about simulation configurations are provided in Table S3 in the supplementary materials.

A shear stress $\tau_{s}$ is applied on the mobile system leading to a total deformation of $0.1 \%$ strain with a constant strain rate of $4.5 \mathrm{~s}^{-1}$, low enough to obtain a quasi-static response using a viscous dislocation mobility law $v(\tau)=\tau . b / B$ (where $B$ is a damping coefficient). Indeed, the dislocation mobility is large enough and nearly isotropic so that the damping constant has no influence on simulations results in the athermal regime. As a consequence, $B$, that is unknown for $\mathrm{UO}_{2}$, is set to $1.510^{-5} \mathrm{~Pa}^{-1}{ }^{-1}$, a sufficiently low value that ensures Forest dislocation interactions to be the rate-limiting process (see section 3.1 of the supplementary information for additional details).

During the simulation, the stress quickly reaches a plateau and fluctuations due to dislocation avalanches and successive junction zipping/unzipping events are observed (see Figure S3 in the supplementary for a typical stress-strain curve). The initial value of the forest density $\rho^{u}=10^{12} \mathrm{~m}^{-2}$ and the stress mean value are used to compute strengthening coefficients using equation (1) $\alpha^{s u}=\sqrt{a^{s u}}=\left\langle\tau^{s}\right\rangle /\left(\mu b \sqrt{\rho^{u}}\right)$, its standard deviation is also provided in the following. Note that dipolar interaction coefficients are not determined using DD model simulations due to the size of the simulation cell, considered too small to provide statistical outputs for such weak interactions.

\section{Results}

\subsection{Dislocation interactions and reaction maps}

Several examples of dislocation reaction maps for $\mathrm{UO}_{2}$ are provided Figure 2. The DD products (junction or annihilation, crossed state and repulsion) depicted by symbols are superimposed to analytical domains computed using the BS and anisotropic approximations (reaction lobes) and the isotropic approximation (borders between attraction and repulsion).

Figure $2 \mathrm{a}, \mathrm{b}$ and c show respectively the reaction maps for the $\operatorname{glissile}_{I / I}^{I I}, \operatorname{sessile}_{I I / I I}^{\{112\}}$ and glissile $I_{I / I I I}^{I I I}$ junctions in $\mathrm{UO}_{2}$. All these junctions have $1 / 2<110>$ Burgers vector but do not lie in the same type of slip plane (see section 2.2). The mean probability to form junctions among all glissile and sessile maps in $\mathrm{UO}_{2}$ is about $41 \%$ (see Table S4.1 and S4.2 
in the supplementary information for the detailed probability of each junction). Here, analytical lobes for the sessile $e_{I I / I I}^{\{112\}}$ and glissile $_{I / I}^{I I}$ are approximately of the same size but DD results show more recombination events for the glissile $I / I$ (42\%) than for the $\operatorname{sessile}_{I I / I I}^{\{112\}}(35 \%)$ reaction. Finally, Figure 2c shows the largest recombination domain (44\%).
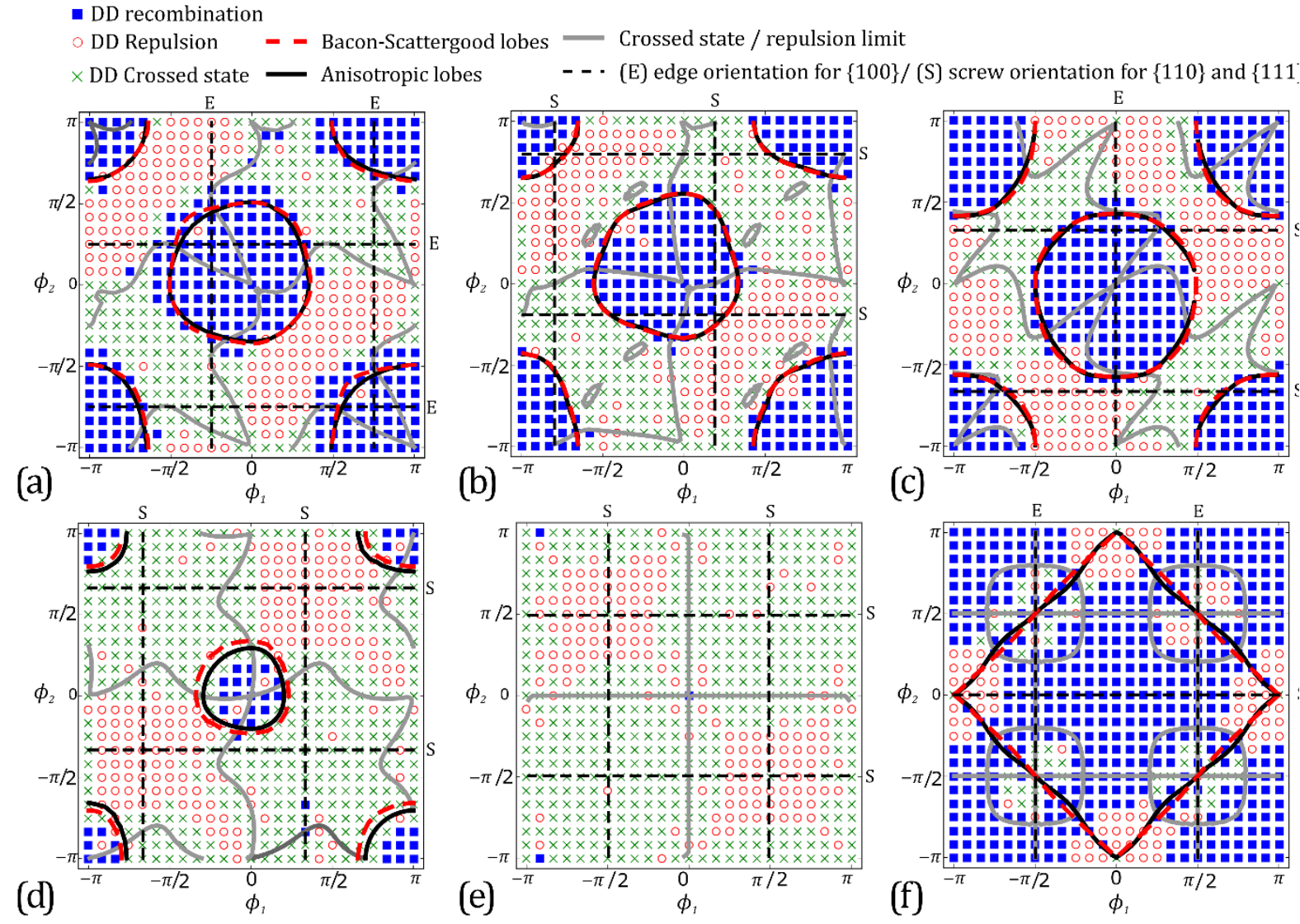

Figure 2: Examples of dislocation reaction maps in $U_{2}$. (a) glissile $e_{I / I}^{I I}\left(\alpha_{2}\right)$ between $A_{I} 1$ and $B_{I} 3$ leading to $E_{I I} 5$ junctions, (b) sessile $e_{I I / I I}^{\{112\}}\left(\alpha_{4}\right)$ between $A_{I I} 1$ with $C_{I I} 3$ forming $1 / 2[1 \overline{1} 0](\overline{1} 12)$ junctions Bacon-Scattergood and anisotropic lobes are superimposed, (c) glissile IIIIIIa $\left(\alpha_{15}\right)$ between $A_{I} 1$ and $A_{I I I} 3$ leading to $B_{I I I} 5$ junctions and, (d) Hirth ${ }_{I I I / I I I}\left(\alpha_{9}\right)$ between $C_{I I I} 1$ and $A_{I I I} 2$ leading to [001](101) junctions, (e) Hirth ${ }_{I I / I I}\left(\alpha_{3}\right)$ between $A_{I I} 1$ and $B_{I I} 2$ leading to $1 / 2[010](100)$ junctions, $(f)$ colinear $_{I / I I}^{90^{\circ}}\left(\alpha_{5}\right)$ between $A_{I} 1$ and $A_{I I} 1$.

The reaction map of the Hirth $I I / I I I$ junction (Figure 2d) is obtained from the reaction between two $1 / 2<110>\{111\}$ parent dislocations with orthogonal Burgers vectors. The junction is sessile with $<100>$ Burgers vector, identical to the FCC Hirth junction which is known to be the weaker among FCC structures. For this junction, the length of the 
junction's Burgers vector does not contribute to reduce the energy. Indeed, the line energy gain arises from the effect of line orientation. As a consequence, the Hirth junction is the most sensitive to the elastic anisotropy [32]. As for the FCC Hirth reaction map, $\mathrm{UO}_{2}$ Hirth $_{I I / I I I}$ exhibits a junction lobe that covers about $8 \%$ of the tested configurations while 92\% lead to crossed and repulsive states.

The Hirth $I I / I I$ reaction map (Figure 2e) shares some crystallographic characteristics

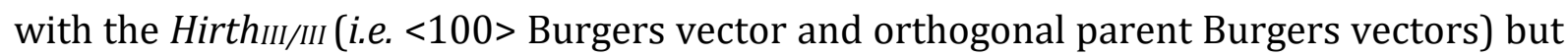
exhibits very different DD results. Indeed, Figure 2e shows only three recombination events (very small junctions) and no analytical recombination domain, whatever the elastic approximations. Moreover, the Hirth ${ }_{I I / I I}$ exhibits one of the lowest recombination rate among all Hirth junctions in $\mathrm{UO}_{2}(\sim 0.5 \%)$. Nevertheless, even with a higher recombination rate, other Hirth maps (except the HirthIII/III) lead mainly to crossed state formations (see Figure S5 in the supplementary) leading to a mean recombination rate of $4 \%$ for the Hirth junctions. This lack of recombination for Hirth maps was previously reported for interaction implying modes I and II using isotropic DD simulation in MgO $[36,40]$.

Finally, Figure $2 \mathrm{f}$ shows the reaction map of the collinear $90_{I / I I}^{9 \circ}$ interaction between $A_{I} 1$, AII 1 slip systems. Here, the recombination rate is about $74 \%$ which is much larger when compared to other reactions. The averaged recombination rate of collinear reactions in $\mathrm{UO}_{2}$ is of $78 \%$.

Discrepancies between the different elastic approaches used are quite small in $\mathrm{UO}_{2}$, especially between BS and full anisotropic calculations and even in cases where the BS approximation is supposed to be less appropriate (Hirth junctions, Figured and e). This is due to the low variation of the Poisson's coefficient ( $\sim 0.33$ with variations of about $10 \%)$ whatever the slip system. As generally observed recombination domains depicted by the analytical solution are in good agreement with DD simulations despite few exceptions like the lobe extension on Figure $2 \mathrm{~b}$ with short junctions obtained using DD simulations in the direct vicinity of the analytical lobe. For collinear reactions, DD results show that annihilations take place in the entire attractive domain as well as in most of the repulsive 
domain (due to the bending of originally repulsive lines that become attractive [48]). Similar flexibility effects are observed especially in junction formation domains.

Based on the previous analysis one can expect that the assumption of elastic isotropy is appropriate for DD computation with $\mathrm{UO}_{2}$ for which the Zener coefficient varies from 0.6 to 0.9 between $1000 \mathrm{~K}$ and $2000 \mathrm{~K}$.

\subsection{Strengthening coefficient model simulations and induced percolation effects}

Table 4: $\mathrm{UO}_{2}$ strengthening coefficient values at $1973 \mathrm{~K}$ for each kind of slip systems combination.

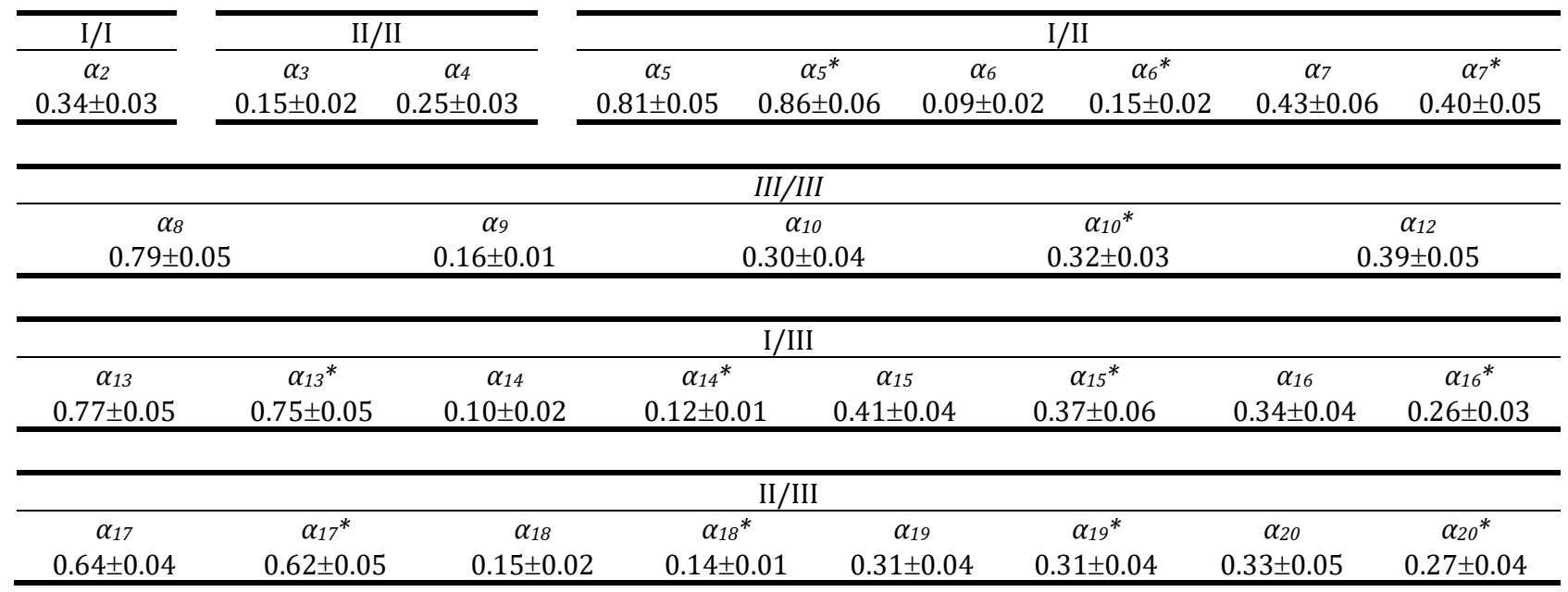

All the strengthening coefficients for $\mathrm{UO}_{2}$ determined by $\mathrm{DD}$ simulations are provided on Table 4. Those coefficients also apply in first approximation to other materials with fluorite (e.g., $\left.\mathrm{CaF}_{2}, \mathrm{BaF}_{2}, \mathrm{ThO}_{2}\right)$ or rock-salt structures (e.g., $\mathrm{NaCl}, \mathrm{MgO}$ or $\mathrm{KCl}$ ) in the absence of a large anisotropy gap. The mean value for coefficient related to glissile junctions is $0.35,0.29$ for sessile ones, 0.13 for Hirth ones and 0.75 for collinear reactions. Coefficient values are in good agreement with the usual hierarchy $[29,32]$ driven by the energy gain during Burgers vector merging and diminution. During the simulations, Frank-Read (F-R) sources show preferred extension directions depending of the investigated interaction. 
Mobile slip system

Forest

junctions

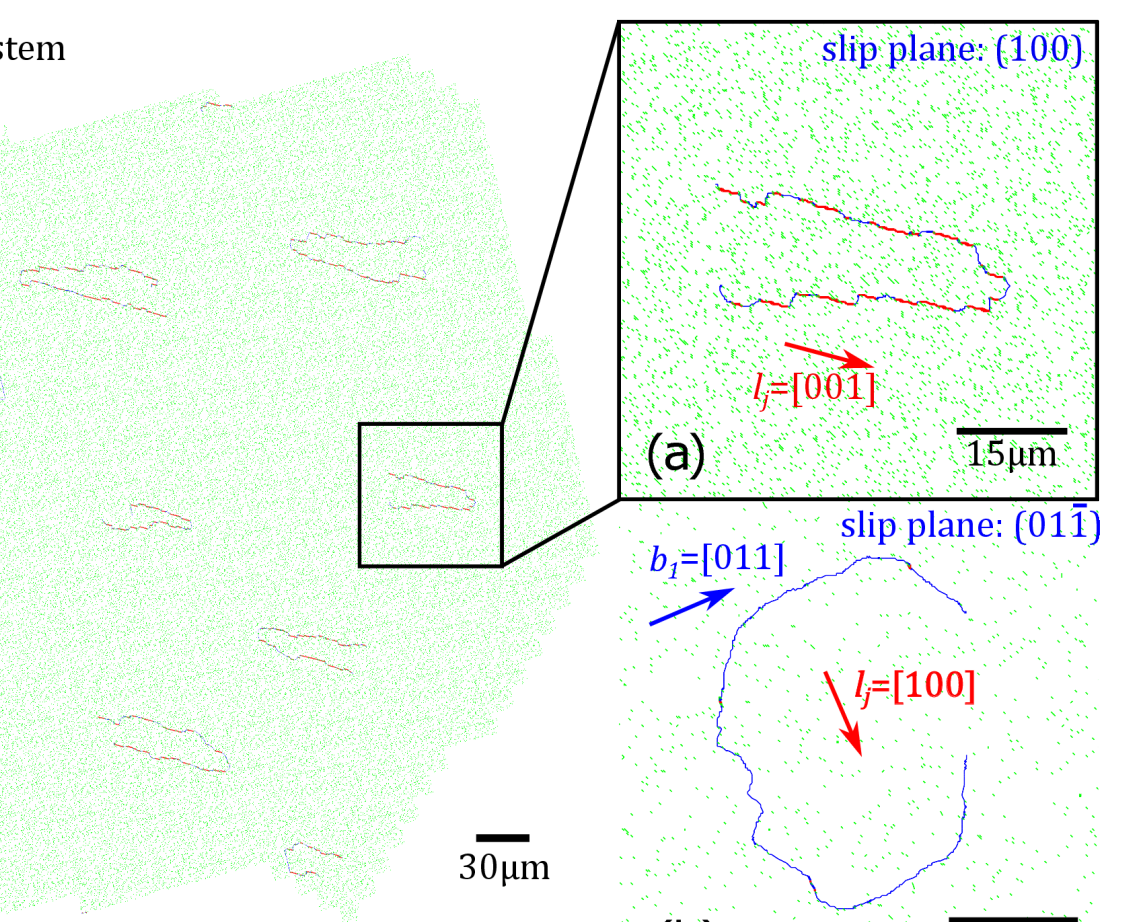

(b)

$15 \mu \mathrm{m}$ slip plane: $(01 \overline{1})$

slip plane: (100)

slip plane: (111)

(c)
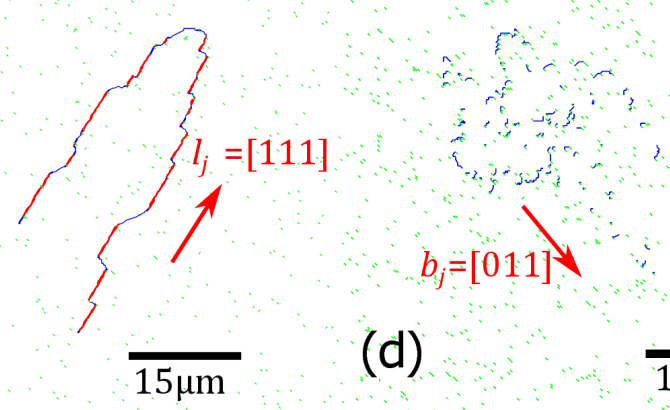

(d)

$15 \mu \mathrm{m}$

(e)

$\overline{15 \mu \mathrm{m}}$

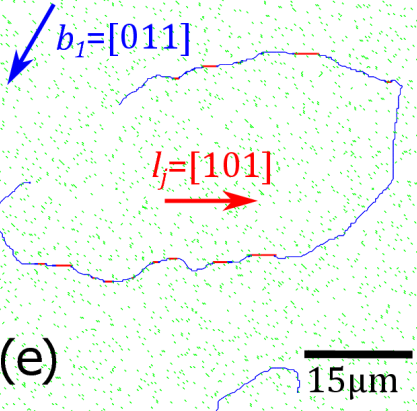

slip plane: $(10 \overline{1})$

(f)

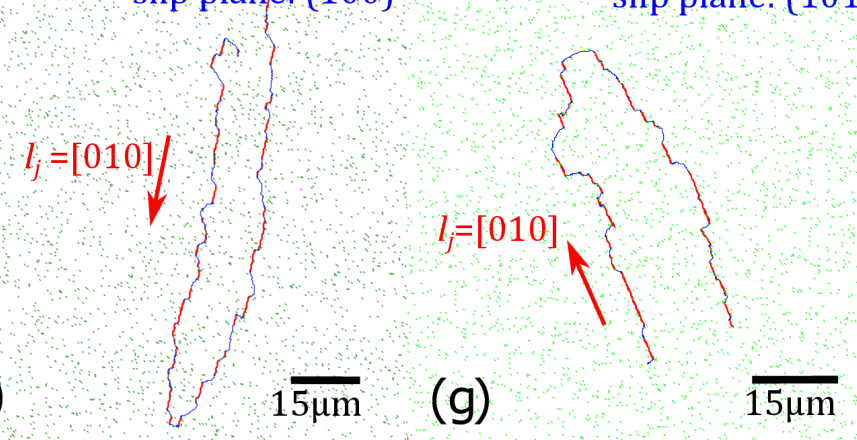


Figure 3: Percolation in the extended slip plane of the mobile slip system during DD model simulation - (a) extended plane $\left(\approx 360 \times 315 \mu \mathrm{m}^{2}\right)$ for coefficient $\alpha_{2}$ with glissile $e_{I / I}^{I I}$ junction and zoom on a dislocation source - (b) zoom for $\alpha_{3}$ with very few and short Hirth ${ }_{I I / I I}$ junctions - (c) zoom of $\alpha_{4}$ with sessile ${ }_{I I / I I}^{\{112\}}$ junctions - (d) zoom of $\alpha_{5}$ with collinear ${ }_{I / I I}^{90^{\circ}}$ reactions - (e) zoom of the $\alpha_{9}$ with original Hirth $_{I I / I I I}$ junctions- (f) zoom of $\alpha_{7}$ with glissile $I_{I / I I}^{I}$ junctions - (g) zoom of $\alpha_{7}{ }^{*}$ with glissile $e_{I I / I}^{I}$ junctions.

Figure 3 shows the percolation of F-R loops thought forest dislocations depending on dislocation reactions during strengthening coefficient model simulations. On the figure, the slip plane of the mobile system is extended through periodic boundary in order to follow dislocation glide on slip distances exceeding widely the box size. Due to the lower energy of the screw dislocation, a free obstacle F-R source is known to be slightly elongated along the screw direction. As shown Figure 3, in the presence of a forest with dislocations from one slip system, the F-R source preferentially progresses along the junction direction leading to very anisotropic shape (see e.g., Figure $3 \mathrm{f}$ for the more anisotropic effect). This result shows that dislocation recombinations act as a driving force to the anisotropic extension of F-R sources. This effect is less obvious in the cases of the Hirth ${ }_{I I / I I}$ junctions (Figure $3 \mathrm{~b}$ ) that are too week to drive a very anisotropic percolations, as shown for stronger obstacles. While few short and weak Hirth II/II junctions are observed, many more crossed-states easily arise along the junction direction (see Figure 2e). This, together with the screw direction which is energetically highly favorable, leads to a more isotropic percolation.

Interaction in $1 / 2<110>\{100\}$ and $1 / 2<110>\{110\}$ slip systems

Three coefficients are derived from symmetries between mode I slip systems: the selfinteraction $\alpha 0^{\prime}$, the dipolar interaction $\alpha_{1}$ and the glissile $e_{I / I}^{I I} \alpha_{2}$. In the case of the glissile $e_{I / I}^{I I}$ model simulation, Figure $3 a$ shows an extension of the F-R source along the [100] direction due to the high probability to make junction (see Figure 2b). Therefore, the associated flow stress during the model simulation is quite high and leads to a coefficient $\alpha_{2}=0.34$. 
Three coefficients are also obtained from $\{110\}$ slip system symmetries including the self-hardening interaction $\alpha_{0}{ }^{\prime}$, and the two sessile junctions i.e., the Hirth ${ }_{I I / I I}$ and a $1 / 2[110]$ stronger sessile $e_{I I / I I}^{\{112\}}$ junction. Only the last two junction coefficients are measured leading to $\alpha_{3}=0.15$ and $\alpha_{4}=0.25$ respectively. These values are quite low for junctions, but agrees with previous calculation of Hirth coefficients in the FCC structure $[29,32]$. Figure $3 b$ shows almost no junctions leading to a F-R loop less elongated along the junction direction than in the case of strong reactions. This result agrees with the interpretation of the dislocation reaction maps (Figure 2e) that shows almost no junction reaction. For the strongest sessile $e_{I I / I I}^{\{112\}}$, a closer analysis revels that the energy gain due to the reaction is the lowest among all $\mathrm{UO}_{2} \frac{1}{2}[110]$ junctions. In addition, the angle between parent dislocations slip planes $\left(\beta_{12}\left(\alpha_{4}\right)=60^{\circ}\right)$ is lower than for the $\operatorname{glissile}_{I / I}^{I I}\left(\beta_{12}\left(\alpha_{2}\right)=90^{\circ}\right)$ leading to less forest crossing events (so a lower number of junctions). However, the percolation shapes observed on Figure $3 \mathrm{a}$ and $\mathrm{c}$ are quite similar showing both coefficients to be associated with a quite strong junctions with a high enough recombination rate (see Figure $2 \mathrm{a}$ and $\mathrm{b}$ ).

Crossed interaction between modes I and II involves three asymmetrical reactions that lead to six coefficients. The collinear $r_{I / I I}^{90^{\circ}}$ interaction is the strongest reaction between slip systems in $\mathrm{UO}_{2}$ and the first case of asymmetrical collinear interaction reported up to now. In this case, the variation of the Poisson's ratios between mobile and forest systems (or, in a more general point of view, the anisotropic energies between the involved slip systems) is the only possible cause for asymmetry. Here, it leads to a significant asymmetry even with a modest variation of Poisson's ratio: $\alpha 5^{*}=0.86$ with mode II mobile slip system $\left(v_{I I}^{B S}=0.32\right)$ while $\alpha_{5}=0.81$ with mode I mobile slip system and $v_{I}^{B S}=0.27$. The high value of the collinear coefficient originates from the wide line energy minimization that happens during the reaction (and the related recombination rate, see Figue $2 \mathrm{f}$ ). $\alpha_{5}$ and $\alpha_{5}{ }^{*}$ exhibit the largest value among all dislocation reactions and even among all collinear interactions [28]. Indeed, the orthogonal slip planes rises the probability for dislocation crossing what increases the annihilation coefficient [32]. Percolation on Figure $3 \mathrm{~d}$ shows that the mobile dislocations annihilate most of the time they cross a forest dislocation cutting them up in small dislocation debris. Finally, the remaining mobile dislocations segments are so small that the related critical stress to initiate the 
movement becomes very high when compared to the critical stress of the strongest junction.

A second reaction between I/II is the HirthI/II that is weaker than the HithII/II including almost no junction formation. So, in the absence of junctions, this coefficient is mainly due to the crossed states formed and will be referred as a crossed state coefficient in the following. A clear asymmetry effect is nevertheless observed with a widely stronger coefficient when mobile system is associated with both the highest Poisson's ratio and a slip planes intersection closer to the screw orientation for mobile dislocations $\left(v_{I}^{B S}=0.27\right.$ and $\beta_{1}=90^{\circ}: \alpha_{6}=0.09-v_{I I}^{B S}=0.32$ and $\left.\beta_{1}=0^{\circ}: \alpha_{6}{ }^{*}=0.14\right)$.

It has been reported that coefficients are stronger when the character of the mobile dislocations is closer to the screw orientation. This effect is related to highly anisotropic percolation, especially in the case of large Poisson's ratio when the line tension makes screw dislocation bending harder [32]. Here, the effect is supposed to be strong as the slip system permutation implies a switch between edge and screw characters. In case of weak reaction, the percolation is not only along the slip plane intersection but also along the screw orientation (when it doesn't match the first direction) leading to a more isotropic and bidirectional geometry. Still, the bidirectional percolation influences the mean line tension, especially along the edge direction, leading to the $\alpha_{6} / \alpha_{6}{ }^{*}$ asymmetry. But the asymmetry is expected to be less pronounced and here it is very strong (see Figure S6.2). The reason why is that very few junctions are formed with a mode I primary system during the model simulation while with a mode II system the number of junctions is nearly the greatest (ten times more, see also Figure S5).

Finally, the last coefficient among I/II interactions is the glissile $I_{I / I I}^{I}$ associated with the coefficients $\alpha_{7}=0.43$ and $\alpha_{7}^{*}=0.40$ both exhibiting a very anisotropic percolation (see Figure $3 \mathrm{f}$ and g). The glissile ${ }_{I / I I}^{I}$ is the strongest junction in $\mathrm{UO}_{2}$ with the largest junction coefficient $\alpha_{7}$, due to the highest probability to form junctions among all $\mathrm{UO}_{2}$ junctions (see table S4.1 in supplementary). $\alpha_{7}^{*}$ is smaller with the mobile slip system from mode I involving the edge character $\left(\beta_{1}\left(\alpha_{7^{*}}\right)=90^{\circ}\right)$. However, this effect is supposed to be narrow as the Poisson's ratio is also smaller for mode I.

Interaction with $1 / 2<110>\{111\}$ slip systems 
The coefficients obtained for mode III reactions are in the same range than the values published for FCC metals $[29,32]$. Those coefficients are usually referenced as $\alpha_{\text {self }}=\alpha 0$, $\alpha_{\text {copla }}=\alpha_{11}, \alpha_{\text {ortho }}=\alpha_{9}, \alpha_{c o l i}=\alpha_{8}, \alpha_{2}=\alpha_{G 0^{\circ}}=\alpha_{10^{*}}\left(\alpha_{G 60^{\circ}}=\alpha_{10}\right)$ and $\alpha_{3}=\alpha_{12}$ in case of FCC metals. The Table 5 provides the comparison of $\mathrm{UO}_{2}$ coefficients with the more recent FCC coefficients with the closest Poisson's ratio i.e. Germanium (Ge) and Aluminum (Al) [32]. The mean values for $\mathrm{UO}_{2}$ are not systematically between the values of $\mathrm{Ge}$ and $\mathrm{Al}$, but a good accordance is obtained.

Table 5: $\mathrm{UO}_{2}$ coefficient values for interaction between systems of $\{111\}$ slip mode compared to Germanium and Aluminum with FCC structure.

\begin{tabular}{lccc}
\hline & $\mathrm{Ge}$ & $\mathrm{UO}_{2}$ & $\mathrm{Al}$ \\
$v$ & 0.25 & 0.30 & 0.36 \\
\hline collinear $_{I I I / I I I}^{70 \circ}\left(\alpha_{8}\right)$ & $0.77 \pm 0.04$ & $0.79 \pm 0.05$ & $0.81 \pm 0.04$ \\
Hirth $_{I I I / I I I}\left(\alpha_{9}\right)$ & $0.18 \pm 0.01$ & $0.16 \pm 0.01$ & $0.20 \pm 0.01$ \\
glissile $_{I I I / I I I}^{I I I}\left(\alpha_{10}\right)$ & $0.30 \pm 0.015$ & $0.30 \pm 0.04$ & $0.30 \pm 0.015$ \\
glissile $_{I I I / I I I}^{I I I}\left(\alpha_{10} 0^{*}\right)$ & $0.31 \pm 0.015$ & $0.32 \pm 0.03$ & $0.32 \pm 0.015$ \\
glissile $_{I I I / I I I}^{I, L}\left(\alpha_{12}\right)$, Lomer & $0.39 \pm 0.02$ & $0.39 \pm 0.05$ & $0.38 \pm 0.02$ \\
\hline
\end{tabular}

As expected, the collinear ${ }_{I I I / I I I}^{70^{\circ}}$ interaction coefficient is the larger among mode III, followed respectively by Lomer, glissile and Hirth. One can notice that $\alpha_{9}$ is the strongest Hirth coefficient within the fluorite structure. This is due to the ability of the system to effectively make Hirth junctions (i.e., presence of a well-defined junction lobe on the reaction map Figure $2 \mathrm{~d}$ ).

Coefficients between mode III and mode I or II are of special interest as they might play a significant role on the single crystal response of $\mathrm{UO}_{2}[8,12,13,16,17]$. Crossed interaction between modes I and III involves four reactions and eight coefficients because of the asymmetry. The $\alpha_{13}=0.77$ and $\alpha_{13}{ }^{*}=0.75$ coefficients are related to the collinear $r_{I / I I I}^{55^{\circ}}$ reaction. The asymmetry is not significant here. The $\alpha_{14}=0.10$ and $\alpha_{14}{ }^{*}=0.12$ related to Hirth $_{I / I I I}$ exhibits a very low coefficient value. Again, here the asymmetry is due to line orientation effect and Poisson's ratios (respectively $\beta_{1}\left(\alpha_{14}\right)=90^{\circ} v_{I}^{B S}=0.27$ and $\beta_{1}\left(\alpha_{14} *\right)=0^{\circ}$ $v_{I I I}^{B S}=0.30$ ). Finally, for interactions between mode I and III, there are two strong glissile coefficients the glissile IIIIIIa and glissile $I_{I / I I I b}^{I I I}$ both leading to a glissile junction in the $\{111\}$ slip mode. As seen for I/II reactions, the asymmetry leads to smaller glissile 
coefficient when the character of the mobile dislocations is close to the edge character. For glissile $e_{I / I I I b}^{I I I}$ coefficient, $\beta_{1}\left(\alpha_{16}\right)=0^{\circ}$ for the mobile system leads to $\alpha_{16}=0.34$ while $\beta_{1}\left(\alpha_{16} *\right)=60^{\circ}$ leads to smaller coefficient value $\alpha_{16}{ }^{*}=0.26$. For glissile $e_{I / I I I a}^{I I I} \alpha_{15}=0.41$ exhibits a larger value than $\alpha_{15^{*}}=0.37$ while $\beta_{1}\left(\alpha_{15}\right)=90^{\circ}>\beta_{1}\left(\alpha_{15^{*}}\right)=60^{\circ}$. Finally, coefficient $\alpha_{15} *$ seems to not follow the usual coefficient hierarchy ruled by the line energy gain, probably due to its large standard deviation (the largest among all junctions).

The interactions between mode II and III are similar to the latter case with one coefficient of each type (collinear, Hirth, glissile and sessile) that are all asymmetrical. The collinear $r_{I I / I I I}^{35^{\circ}}$ is the weakest among all collinear coefficients in $\mathrm{UO}_{2}\left(\alpha_{17}=0.64\right)$. It has the highest recombination probability $(81 \%$, based on its reaction map that assumes the dislocation lines to cross each other) what it is likely due to the angle between the parent slip planes which is the lowest among all collinear configurations $\left(\beta_{12}\left(\alpha_{17}\right)=35^{\circ}\right)$. This geometry promotes strong dislocation interactions able to bend dislocation lines and force reactions. However, this is not the most important effect of this angle. Indeed, $\beta_{12}$ also drives the crossing probability of dislocation lines: decreasing $\beta_{12}$ lower the crossing probability and the combination of both effects leads to a lower coefficient value. The $\operatorname{Hirth}_{I I / I I I}^{\{012\}}$ has a stronger coefficient for $\alpha_{18}=0.15$ than for $\alpha_{18}{ }^{*}=0.14$ but the variation is not significant due to a low variation of dislocation character and $v^{B S}$ (from $\beta_{1}\left(\alpha_{18}\right)=55^{\circ}$ $v_{I I}^{B S}=0.32$ to $\left.\beta_{1}\left(\alpha_{18^{*}}\right)=90^{\circ} v_{I I I}^{B S}=0.30\right)$. The sessile $e_{I I / I I I}^{\{113\}}$ does not exhibit asymmetry with $\alpha_{19}=\alpha_{19}{ }^{*}=0.31$ due to both the almost neutral character of the parent dislocation for both slip systems $\beta_{1}\left(\alpha_{19}\right)=55^{\circ}$ and $\beta_{1}\left(\alpha_{19^{*}}\right)=30^{\circ}$ and the close Poisson's ratio $\left(v_{I I I}^{B S}=0.30\right.$ and $v_{I I}^{B S}=0.32$ ). Finally, the asymmetry for the glissile $I I I / I I I$ is mainly due to the screw character of the junction for $\alpha_{20}=0.33$ while it is close to edge $\left(\beta_{1}\left(\alpha_{20} 0^{*}\right)=60^{\circ}\right)$ for $\alpha_{20}{ }^{*}=0.27$.

\section{Discussion}




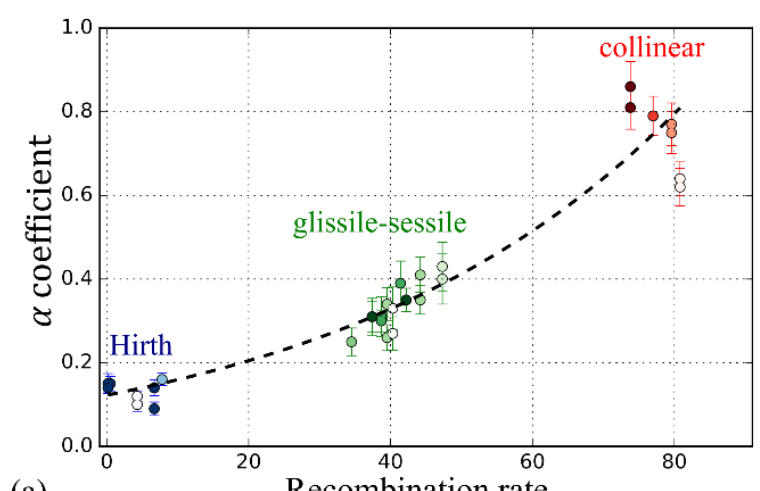

(a)

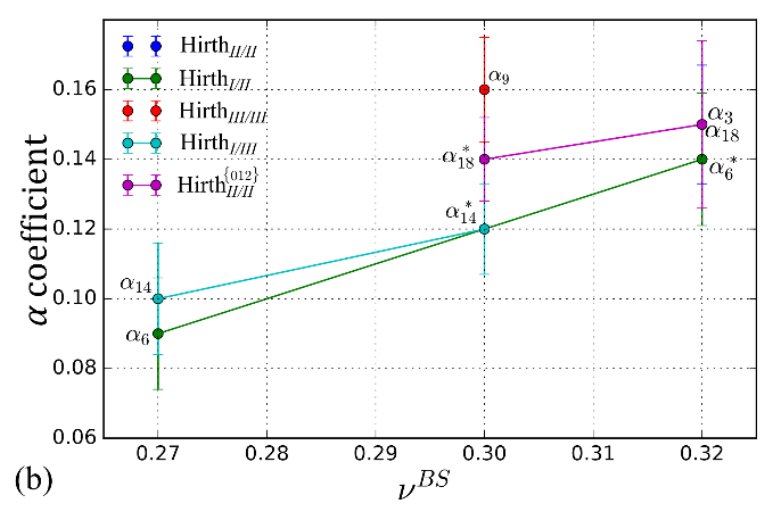

Figure 4: (a) Interaction coefficients versus reaction maps recombination rate. Hirth junctions are in blue, glissile and sessile junctions are in green and collinear reactions are in red. For all reaction groups, darker colors refer to larger $\beta_{12}$ angles. The dashed line is a guide for the eyes. (b) Hirth coefficients versus Poisson's ratio.

As inferred from the results section, the amplitude of a given interaction coefficient depends on (i) the energetical aspect of the contact reactions as described by the reaction maps (i.e., dislocation line, Burgers vector and parent slip planes), (ii) the topology of the dislocation process (percolations) and (iii) the probability for dislocations to intersect which is driven by the angle between slip planes $\left(\beta_{12}\right)$.

Figure 4a exhibits a continuous increase of the interaction coefficient that scales with reaction maps recombination rate leading to three delimited domains relative to Hirth junctions, glissile/sessile junctions and collinear reactions. Interactions coefficients of a given group of contact reactions are colored according to $\beta_{12}$ (the larger $\beta_{12}$ the darker the marker) in order to quantify correlations. While the collinear coefficients continuously increase when increasing $\beta_{12}$ confirming previous observations made in BCC metals [32], Hirth and sessile/glissile coefficients do not scale with $\beta_{12}$. This property is due to the high similarity (in terms of in plane geometry) of collinear reactions that overstates the role of $\beta_{12}$ whereas its impact on junction coefficients is limited by the role of other 
configurational parameters. As an example, the Hirth coefficients rather scale with the Poisson's ratio as shown by Figure $4 \mathrm{~b}$.

Overall, these results confirm the main role of the recombination rate over other configurational parameters such as $\beta_{12}, \beta_{1}$, and, at a lesser extent, $v^{B S}$, that do not lead to comparable correlations (see section S6 in the supplementary for more details).

Based on the analysis of dislocation interaction coefficients, one can derive a qualitative description of strain-hardening in $\mathrm{UO}_{2}$ single crystal. Indeed, data from Table 4 show that strain hardening within individual modes I and II should be moderately high, as characterized by dislocation reaction coefficients of intermediate amplitude (respectively, $\alpha_{2}=0.34$ and $\alpha_{3}=0.15, \alpha_{4}=0.25$ ). On the other hand, an enhanced strengthening effect should be noticed in case of simultaneous activation of modes I and II as shown by larger interaction coefficients values (e.g., collinear reactions with coefficients of about $\alpha_{5}=0.81$ ).

This hypothesis was first discussed in Amodeo et al. [36], in the case of Mg0, that also exhibits $1 / 2<110>\{110\}$ and $1 / 2<110>\{100\}$ slip systems as main slip modes. In Amodeo et al. [36], the authors used isotropic DD simulations to investigate MgO single crystal deformation with compression axis (CA) along $<100>$ and $<110>$. Only single mode simulations were carried. In the case of CA aligned with $\langle 100\rangle$, the authors considered dislocations in $1 / 2<110>\{110\}$ slip systems only, including four over six activated slip systems (the same dislocation density was set on each slip system). On the other hand, additional simulations accounting for $1 / 2<110>\{100\}$ dislocations with four over six slip systems activated with CA aligned with $<110>$ were performed. The authors derived

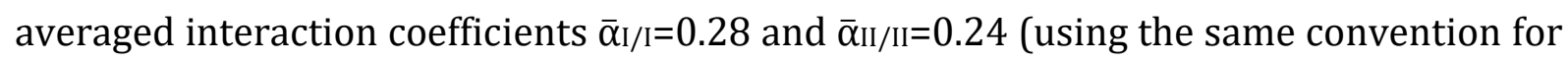
mode I and II than here for $\mathrm{UO}_{2}$ ) from stress-strain and dislocation density evolutions. These averaged coefficients rely on all possible interactions occurring in each mode.

For $\mathrm{UO}_{2}$, similar averaged coefficients for mode I and II can be defined using the same relative orientations that in Amodeo et al. [36] and the following equations:

$$
\bar{\alpha}_{\mathrm{I} / \mathrm{I}}=\sqrt{\left(2 a_{2}+a_{1}+a_{0}{ }^{\prime}\right) / 4}
$$

and 


$$
\bar{\alpha}_{I I / I I}=\sqrt{\left(2 a_{4}+a_{3}+a_{0}{ }^{\prime}\right) / 4}
$$

In this approach, only interactions between activated slip systems are considered as they are usually characterized by a larger obstacle dislocation density (due to dislocation multiplication) than slip systems with zero Schmid factor. Assuming a negligible effect of elastic coefficients variations between $\mathrm{MgO}$ and $\mathrm{UO}_{2}$ (Amodeo et al. used $v=0.33$ on both slip modes), one can easily derive the self-hardening coefficient $\alpha_{0}{ }^{\prime}=\sqrt{a_{0}{ }^{\prime}}=0.29$ and the dipolar coefficient $\alpha_{1}$ which is null (indeed dipolar interactions are negligible in comparison to junction effects), using both $\mathrm{MgO}$ and $\mathrm{UO}_{2}$ coefficients in equations (2) and (3). While they remain qualitative, these coefficients are in good agreement with those expected from the literature. Indeed, $\alpha_{0}{ }^{\prime}$ can directly be compared to the value calculated in $\mathrm{Cu}\left(\alpha 0^{\prime}=0.35\right)$ [39] while less than 0.1 is expected for dipolar interactions [34].

One can extend this approach integrating the influence of the crystal orientation as shown Figure 5 using inverse pole figures (IPF). While this approach is partially qualitative due to the thermal/athermal character of some deformation modes in $\mathrm{UO}_{2}\left(T_{a}^{I}\right.$ ranges from $1400 \mathrm{~K}$ to $1750 \mathrm{~K}[10,16], T_{a}^{I I}>2000 \mathrm{~K}[16]$ and no information about $T_{a}^{I I I}$ ), we will show that it allows to easily lay the foundation of a strain-hardening model for $\mathrm{UO}_{2}$ single crystal at high-temperature.

Averaged coefficients $\bar{\alpha}$ for individual and pairs of modes are calculated using equation (4). As a criterion for plasticity, slip systems with Schmid factor $m_{k}$ larger than 0.15 are considered together with those characterized by $m_{k}>0.8 m_{k}^{\max }$ (with $m_{k}^{\max }$ the maximum Schmid factor).

$$
\begin{gathered}
\bar{\alpha}=\sqrt{\frac{\sum_{s=1}^{N} \delta_{s} \sum_{u}^{N} \delta_{u} a^{s u}}{\sum_{s=1}^{N} \delta_{s} \sum_{u=1}^{N} \delta_{u}}} \\
\delta_{k}=1 \text { if } m_{k} \geq 0.15 \text { and } m_{k} \geq 0.8 m_{k}^{\max } \\
\text { else } \delta_{k}=0
\end{gathered}
$$

Figure 5 shows that $\bar{\alpha}_{I / I}$ reach intermediate values for most of the orientations what promotes $1 / 2<110>\{100\}$ slip system activation in $\mathrm{UO}_{2}$ single crystal except close to [001] where $m_{\{100\}}$ is null (Figure $5 \mathrm{a}$ ). Strengthening is also quite homogenous but slightly softer for mode II (Figure 5b). In this last case, the weak activation of mode II close to [1111] also 
enhances mode I activation for these orientations whereas mode II slip systems should be favored nearby [001]. As commonly observed in FCC, mode III strengthening is particularly high [32] with $\bar{\alpha}$ ranging from 0.20 close to [122] up to 0.42 along [111] (Figure 5c).

Crossed interactions between modes I and II (Figure 5d) induce strengthening for orientations in the central domain of the IPF where both modes benefit of activated slip systems verifying equation (4) leading to several collinear interaction configurations. Nonetheless, this domain is particularly narrow and white regions are typical of orientations where slip systems with higher Schmid factors are restricted to a unique slip mode (I or II for white region in the vicinity of [001] and [111] respectively). This additional strengthening induced by modes I and II common activation (Figure 5d) is in good agreements with single crystal experiments of Sawbridge and Sykes [8], for which stress-strain curves show a particularly high strengthening rate for orientation $\phi$ ranging from $25^{\circ}$ to $36^{\circ}$ only. The lack of crossed interaction close to [011] $\left(\Omega=0^{\circ}\right)$ also confirms the weak contribution of mode II for theses orientations as suggested by Portelette et al. [16].

Interactions I/III (Figure 5e) could also contribute to the strain-hardening observed on experimental stress-strain curves. They are particularly high $\left(\bar{\alpha}_{I / I I I}=0.60\right)$ in a domain in between [111] and [011]. Finally, dislocation interactions between mode III and II lead to $\bar{\alpha}_{I I I / I I}$ ranging from 0.38 to 0.45 (Figure $5 f$ ).

Assuming the controversial aspect of mode III dislocations in $\mathrm{UO}_{2}$, one could expect a significantly different mechanical response in case of dislocation multiplication in the three modes when compared to a simpler model that accounts only for modes I and II. Interestingly, III/III interactions strongly strengthen mode III in regions where mode I and II are dominant in terms of slip activity (mode II near [001] with $m_{\{110\}}^{\max }=0.5$ and mode I near [111] with $m_{\{100\}}^{\max }=0.47$ respectively) but exhibit weak interactions. In addition, crossed interactions between mode III and II (Figure 5f) with high value of $\bar{\alpha}_{I I I / I I}$ near [001] do not promote activation of mode III in this zone while Figure 5e shows that Schmid factor is too unfavorable for mode III activation near [111]. Therefore, mode III crossed contribution is most likely expected in the central part of the IPF where it spans a larger zone than cross interaction between modes I and II. 
This is of special interest as Sawbridge and Sykes observed softening or no hardening on stress-strain curves for $\phi<25^{\circ}$ and $\phi>36^{\circ}$ suggesting a low dislocation multiplication rate in mode III for these orientations.

Consequently, the low strain-hardening observed for $\phi<25^{\circ}$ in Sawbridge experiments could be attributed to the moderate values of $\bar{\alpha}_{I / I}, \bar{\alpha}_{I I / I I}$ and $\bar{\alpha}_{I / I I}$ and the lack of dislocations in $1 / 2<110>\{111\}$. Moreover, Sawbridge and Sykes also observed hardening in the middle of the mode I/II interaction zone (Figure $5 \mathrm{~d}$ ) but not near [011]. This agrees with $\bar{\alpha}_{I / I I}$ values but not with $\bar{\alpha}_{I / I I I}$ near [011] what suggests again a lack of dislocations in $1 / 2<110>\{111\}$, at least near [011].

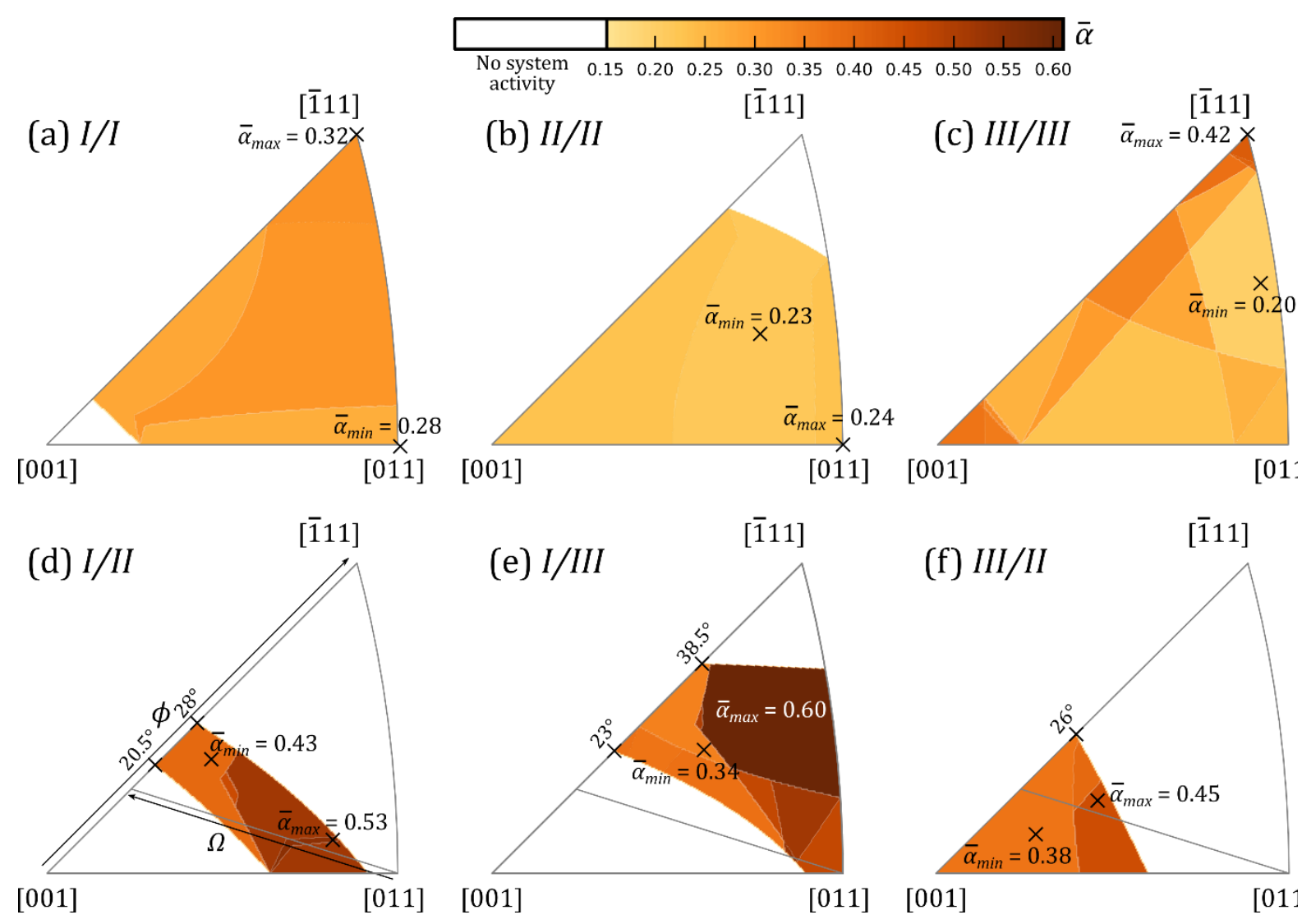

Figure 5 : IPFs showing the evolution of the averaged dislocation interaction coefficients for (a) I/I, (b) II/II, (c) III/III, (d) I/II, (e) I/III and (f) III/II mode interactions computed using equation (4) for various orientations. For crossed interactions, the lack of strengthening is related to low Schmid factors in mode I slip systems for the left part of (d) and (e), in mode II in the upper and right part of (f) and in mode III for the upper part of (e).

As previously discussed, modes II and III should be characterized by partial and/or high lattice friction what does not fit the forest model used here. However, results 
presented Figure 5 already provide some hints about $\mathrm{UO}_{2}$ single crystal deformation under incidental conditions of temperature. First Figure $5 \mathrm{a}, \mathrm{b}$ and $\mathrm{c}$ confirm the crucial role of I/I and I/II dislocation interactions that are configurations mostly athermal. This is particularly true for I/I configurations and should be investigated for I/II as the curvature of dislocations in mode I slip systems could lead to the formation of several junctions, even if crossing dislocation from slip systems characterized by lattice friction (such as mode II in these conditions of temperature). Interactions II/II are mostly weaker, and III/III are only partially required to explain strain-hardening observed in the experiments.

This study addresses a detailed description of dislocation interactions and strengthening reactions in $\mathrm{UO}_{2}$ required to build first physics-based model for strain hardening in in $\mathrm{UO}_{2}$ single crystal. Nonetheless, dislocation microstructural aspects such as the dislocation density spread per slip mode or a detailed analysis of mode III contribution still need to be investigated, e.g., using transmission electron microscopy, to clarify their role among $\mathrm{UO}_{2}$ single-crystal mechanical properties within the incidental conditions.

\section{Conclusions}

Here are resumed the main conclusions of the study:

- Dislocation Dynamics simulations are used to investigate the strengthening elementary processes, dislocation reaction maps and compute Franciosi's interaction matrix coefficients for the three deformation modes $1 / 2<110>\{100\}$ (mode I), $1 / 2<110>\{110\}$ (mode II) and $1 / 2<110>\{111\}$ (mode III) of $\mathrm{UO}_{2}$.

- The interaction coefficients scales with the recombination rate and parameters such as the angle between parent slip modes and the Poisson's ratio have shown to influence particular reactions such as collinear interactions and Hirth's junctions respectively.

- When only one slip-mode is activated, modes I and II are characterized by intermediate dislocation coefficients, while those of mode III are larger. Largest 
values are also obtained when two modes are activated together (e.g., modes I and II) especially in the case of strong collinear dislocation interactions with coefficient that can exceed 0.8 .

- Averaged strengthening coefficients are presented and their variations with the sample orientation are discussed. Results are in good agreement with Sawbridge and Sykes [8] experiments.

- Such coefficients can be used in macroscopic models to describe the deformation induced by dislocation glide in $\mathrm{UO}_{2}$ at high-temperature.

\section{Acknowledgements}

This research is achieved in the framework of a simulation project devoted to the PLEIADES fuel software environment funding by the French nuclear institute between CEA, EDF and FRAMATOME. The authors acknowledge S. Loridon who developed the full anisotropic lobe calculation procedure.

\section{References}

[1] B. Michel, C. Nonon, J. Sercombe, F. Michel, V. Marelle, Simulation of Pellet-Cladding Interaction with the Pleiades Fuel Performance Software Environment, Nucl. Technol. 182 (2013) 124-137. https://doi.org/10.13182/NT13-A16424.

[2] B. Michel, J. Sercombe, G. Thouvenin, A new phenomenological criterion for pelletcladding interaction rupture, Nucl. Eng. Des. 238 (2008) 1612-1628. https://doi.org/10.1016/j.nucengdes.2008.01.012.

[3] R.L. Williamson, K.A. Gamble, D.M. Perez, S.R. Novascone, G. Pastore, R.J. Gardner, J.D. Hales, W. Liu, A. Mai, Validating the BISON fuel performance code to integral LWR experiments, Nucl. Eng. Des. $301 \quad$ (2016) 232-244. https://doi.org/10.1016/j.nucengdes.2016.02.020.

[4] E.J. Rapperport, A.M. Huntress, Deformation Modes of Single Crystal Uranium Dioxide from $700^{\circ} \mathrm{C}$ to $1900^{\circ} \mathrm{C},(1960)$. 
[5] J.F. Byron, The yield and flow of single crystals of uranium dioxide, J. Nucl. Mater. 28 (1968) 110-114. https://doi.org/10.1016/0022-3115(68)90062-7.

[6] J.S. Nadeau, Dependence of Flow Stress on Nonstoichiometry in Oxygen-Rich Uranium Dioxide at High Temperatures, J. Am. Ceram. Soc. 52 (1969) 1-. https://doi.org/10.1111/j.1151-2916.1968.tb11863.x-i1.

[7] C.S. Yust, C.J. McHargue, Dislocation substructures in deformed uranium dioxide single crystals, J. Nucl. Mater. 31 (1969) 121-137. https://doi.org/10.1016/00223115(69)90187-1.

[8] P.T. Sawbridge, E.C. Sykes, Dislocation Glide in UO2 Single Crystals at $1600^{\circ} \mathrm{K}$, Philos. Mag. 24 (1971) 33-53.

[9] M.S. Seltzer, A.H. Clauer, B.A. Wilcox, The influence of stoichiometry on compression creep of uranium dioxide single crystals, J. Nucl. Mater. 44 (1972) 43-56. https://doi.org/10.1016/0022-3115(72)90127-4.

[10] J.M. Lefebvre, Contribution à l'étude de la déformation plastique d'une céramique de strucuture fluorite : le bioxyde d'uranium, Faculté des sciences de poitiers, 1976.

[11] A. Alamo, J.M. Lefebvre, J. Soullard, Deformation plastique du bioxyde d'uranium: Observation des sous-structures de dislocations, J. Nucl. Mater. 75 (1978) 145-153. https://doi.org/10.1016/0022-3115(78)90038-7.

[12] R.J. Keller, T.E. Mitchell, A.H. Heuer, Plastic deformation in nonstoichiometric UO2+x single crystals-I. Deformation at low temperatures, Acta Metall. 36 (1988) 10611071. https://doi.org/10.1016/0001-6160(88)90160-5.

[13] R.J. Keller, T.E. Mitchell, A.H. Heuer, Plastic deformation in nonstoichiometric UO2 + x single crystals-II. Deformation at high temperatures, Acta Metall. 36 (1988) 1073-1083. https://doi.org/10.1016/0001-6160(88)90161-7.

[14] A.V. Lunev, A.Y. Kuksin, S.V. Starikov, Glide mobility of the 1/2[110](001) edge dislocation in UO2 from molecular dynamics simulation, Int. J. Plast. 89 (2017) 8595. https://doi.org/10.1016/j.ijplas.2016.11.004.

[15] A. Soulié, J.P. Crocombette, A. Kraych, F. Garrido, G. Sattonnay, E. Clouet, Atomistically-informed thermal glide model for edge dislocations in uranium 
dioxide, Acta $\quad$ Mater. $150 \quad$ (2018) 248-261. https://doi.org/10.1016/j.actamat.2018.03.024.

[16] L. Portelette, J. Amodeo, R. Madec, J. Soulacroix, T. Helfer, B. Michel, Crystal viscoplastic modeling of U02 single crystal, J. Nucl. Mater. (2018). https://doi.org/10.1016/j.jnucmat.2018.06.035.

[17] P. Fossati, L. Van Brutzel, B. Devincre, Molecular dynamics simulation of dislocations in uranium dioxide, J. Nucl. Mater. 443 (2013) 359-365. https://doi.org/10.1016/j.jnucmat.2013.07.059.

[18] R. Skelton, A.M. Walker, Peierls-Nabarro modeling of dislocations in U02, J. Nucl. Mater. 495 (2017) 202-210. https://doi.org/10.1016/j.jnucmat.2017.08.024.

[19] A.V. Lunev, S.V. Starikov, T.N. Aliev, V.I. Tseplyaev, Understanding thermallyactivated glide of $1 / 2\langle 110\rangle\{110\}$ screw dislocations in UO2 - A molecular dynamics analysis, Int. J. Plast. $110 \quad$ (2018) 294-305. https://doi.org/10.1016/j.ijplas.2018.07.003.

[20] P. Franciosi, A. Zaoui, Université de Paris-Nord, Etude théorique et expérimentale du comportement élastoplastique des monocristaux métalliques se déformant par glissement modélisation pour un chargement complexe quasi statique, [s.n.], 1984.

[21] G.I. Taylor, The Mechanism of Plastic Deformation of Crystals. Part I. Theoretical, Proc. R. Soc. Lond. Math. Phys. Eng. Sci. 145 (1934) 362-387. https://doi.org/10.1098/rspa.1934.0106.

[22] G. Saada, Sur le durcissement dû à la recombinaison des dislocations, Acta Metall. 8 (1960) 841-847. https://doi.org/10.1016/0001-6160(60)90150-4.

[23] B. Devincre, L.P. Kubin, Simulations of forest interactions and strain hardening in FCC crystals, Model. Simul Mater Sci Eng. 2 (1994) 559-570. https://doi.org/10.1088/0965-0393/2/3A/010.

[24] B. Devincre, R. Madec, G. Monnet, S. Queyreau, R. Gatti, L.P. Kubin, Modeling crystal plasticity with dislocation dynamics simulations: the 'microMegas' code, Mech. Nano-Objects. (2011) 81-100.

[25] V.V. Bulatov, L.L. Hsiung, M. Tang, A. Arsenlis, M.C. Bartelt, W. Cai, J.N. Florando, M. Hiratani, M. Rhee, G. Hommes, T.G. Pierce, T.D. de la Rubia, Dislocation multi- 
junctions and strain hardening, Nature. 440 (2006) 1174-1178. https://doi.org/10.1038/nature04658.

[26] W. Cai, V.V. Bulatob, J. Chang, J. Li, S. Yip, Periodic image effects in dislocation modelling, Philos. Mag. $83 \quad$ (2003) 539-567. https://doi.org/10.1080/0141861021000051109.

[27] R. Madec, B. Devincre, L.P. Kubin, On the use of periodic boundary conditions in dislocation dynamics simulations, Springer, Dordrecht, 2004.

[28] R. Madec, B. Devincre, L.P. Kubin, T. Hoc, D. Rodney, The Role of Collinear Interaction in Dislocation-Induced Hardening, Science. 301 (2003) 1879-1882. https://doi.org/10.1126/science.1085477.

[29] B. Devincre, L.P. Kubin, T. Hoc, Physical analyses of crystal plasticity by DD $\begin{array}{lllll}\text { simulations, } & \text { Scr. } & \text { Mater. } & 54 & \text { (2006) }\end{array}$ https://doi.org/10.1016/j.scriptamat.2005.10.066.

[30] A. Alankar, I.N. Mastorakos, D.P. Field, H.M. Zbib, Determination of Dislocation Interaction Strengths Using Discrete Dislocation Dynamics of Curved Dislocations, J. Eng. Mater. Technol. 134 (2012) 021018-021018-4. https://doi.org/10.1115/1.4005917.

[31] N. Bertin, C.N. Tomé, I.J. Beyerlein, M.R. Barnett, L. Capolungo, On the strength of dislocation interactions and their effect on latent hardening in pure Magnesium, Int. J. Plast. 62 (2014) 72-92. https://doi.org/10.1016/j.ijplas.2014.06.010.

[32] R. Madec, L.P. Kubin, Dislocation strengthening in FCC metals and in BCC metals at high temperatures, Acta Mater. 126 (2017) 166-173. https://doi.org/10.1016/j.actamat.2016.12.040.

[33] R. Madec, L.P. Kubin, Dislocation dynamics in BCC metals: interaction strengths in the athermal regime, in: Comput. Model. Simul. Mater. III - Part A, Advances in Science and Technology, Techna Group srl, 2004: pp. 671-678. https://www.researchgate.net/publication/258242352_Dislocation_dynamics_in_ BCC_metals_interaction_strengths_in_the_athermal_regime/stats. 
[34] S. Queyreau, G. Monnet, B. Devincre, Slip systems interactions in $\alpha$-iron determined by dislocation dynamics simulations, Int. J. Plast. 25 (2009) 361-377. https://doi.org/10.1016/j.ijplas.2007.12.009.

[35] B. Devincre, Dislocation dynamics simulations of slip systems interactions and forest strengthening in ice single crystal, Philos. Mag. 93 (2013) 235-246. https://doi.org/10.1080/14786435.2012.699689.

[36] J. Amodeo, B. Devincre, Ph. Carrez, P. Cordier, Dislocation reactions, Plastic anisotropy and forest strengthening in $\mathrm{MgO}$ at high temperature, Mech. Mater. 71 (2014) 62-73. https://doi.org/10.1016/j.mechmat.2014.01.001.

[37] J. Durinck, B. Devincre, L. Kubin, P. Cordier, Modeling the plastic deformation of olivine by dislocation dynamics simulations, Am. Mineral. 92 (2007) 1346-1357. https://doi.org/10.2138/am.2007.2512.

[38] E. Schmid, W. Boas, Kristallplastizität: Mit Besonderer Berücksichtigung der Metalle, $\begin{array}{lll}\text { Springer-Verlag, } & \text { Berlin } & \text { Heidelberg, }\end{array}$ https://www.springer.com/gp/book/9783662342619 (accessed October 9, 2019).

[39] B. Devincre, L.P. Kubin, T. Hoc, Collinear superjogs and the low-stress response of fcc $\begin{array}{lllll}\text { crystals, } & \text { Scr. } & \text { Mater. } & 57 & \text { (2007) }\end{array}$ https://doi.org/10.1016/j.scriptamat.2007.07.026.

[40] P. Carrez, P. Cordier, B. Devincre, L.P. Kubin, Dislocation reactions and junctions in MgO, Mater. Sci. Eng. -Struct. Mater. Prop. Microstruct. Process. 400 (2005) 325-328. https://doi.org/10.1016/j.msea.2005.03.071.

[41] R. Madec, B. Devincre, L.P. Kubin, On the nature of attractive dislocation crossed states, Comput. Mater. Sci. 23 (2002) 219-224. https://doi.org/10.1016/S09270256(01)00215-4.

[42] R. Madec, L.P. Kubin, Second-order junctions and strain hardening in bcc and fcc crystals, Scr. $\quad$ Mater. 58 (2008) 767-770. https://doi.org/10.1016/j.scriptamat.2007.12.032.

[43] W. Puschl, Reactions Between Glide Dislocations and Forest Dislocations in Anisotropic Bcc Metals, Phys. Status Solidi -Appl. Res. 90 (1985) 181-189. https://doi.org/10.1002/pssa.2210900117. 
[44] R. Madec, B. Devincre, L.P. Kubin, From Dislocation Junctions to Forest Hardening, $\begin{array}{lllll}\text { Phys. } & \text { Rev. } & \text { Lett. } & 89 & \text { (2002) }\end{array}$ https://doi.org/10.1103/PhysRevLett.89.255508.

[45] J. Douin, DisDi, (n.d.). http://joel.douin.free.fr/Disdi-Page.html.

[46] J. Douin, P. Veyssiere, P. Beauchamp, Dislocation line stability in Ni3AI, Philos. Mag. A. 54 (1986) 1375-393.

[47] M.T. Hutchings, High-temperature studies of UO2 and ThO2 using neutron scattering techniques, J. Chem. Soc. Faraday Trans. 2 Mol. Chem. Phys. 83 (1987) 1083-1103. https://doi.org/10.1039/F29878301083.

[48] L.P. Kubin, R. Madec, B. Devincre, Dislocation intersections and reactions in FCC and BCC crystals, in: H.M. Zbib, D.H. Lassila, L.E. Levine, K.J. Hemker (Eds.), Multiscale Phenom. Mater.-Exp. Model. Relat. Mech. Behav., Materials Research Society, Warrendale, 2003: pp. 25-36. 


\title{
Supplementary Information
}

\section{Athermal dislocation strengthening in $\mathrm{UO}_{2}$}

\author{
Luc Portelette ${ }^{1}$, Jonathan Amodeo ${ }^{2}$, Bruno Michel ${ }^{1}$, Ronan Madec ${ }^{3}$
}

1 CEA, DEN, DEC, SESC, LSC bat 151 Centre de Cadarache F-13108 Saint Paul Lez Durance, France

2 Université de Lyon, INSA-Lyon, CNRS, MATEIS UMR5510, F-69621 Villeurbanne, France

${ }^{3}$ CEA, DAM, DIF, F-91297 Arpajon, France

\section{Supplementary Section 1}

\section{Crystallography and symmetries}

Table S1: Interaction matrix for $\mathrm{UO}_{2}$. Each line represents a mobile system, each column a forest system and each cell a coefficient. Coefficients with stars are asymmetrical coefficient.

\begin{tabular}{|c|c|c|c|c|c|c|c|c|c|c|c|c|c|c|c|c|c|c|c|}
\hline & \multirow{2}{*}{\begin{tabular}{|c|}
$\mathrm{I}$ \\
$\mathrm{A} 1 \mathrm{~A} 2 \mathrm{~B} 3 \mathrm{~B} 4 \mathrm{C} 5 \mathrm{C} 6$ \\
\end{tabular}} & \multicolumn{6}{|c|}{ II } & \multicolumn{12}{|c|}{ III } \\
\hline & & A1 & B2 & $\mathrm{C} 3$ & $\mathrm{D}$ & כ & F6 & C1 & & & & & C3 & B4 & & 35 & $\mathrm{C} 5$ & A6 & 6 \\
\hline A1 & $\begin{array}{llllll}a_{0} & a_{1} & a_{2} & a_{2} & a_{2} & a_{2}\end{array}$ & $a_{5}$ & $a_{6}$ & $a_{7}$ & $a_{7}$ & $a_{7}$ & $a_{7}$ & $a_{13}$ & 13 & 114 & 114 & 15 & $a_{16}$ & $a_{15}$ & $a_{16}$ & 915 & $a_{16}$ & $a_{15}$ & 916 \\
\hline A2 & $\begin{array}{llllll}a_{1} & a_{0} & a_{2} & a_{2} & a_{2} & a_{2}\end{array}$ & $a_{6}$ & $a_{5}$ & $a_{7}$ & $a_{7}$ & $a_{7}$ & $a_{7}$ & $a_{14}$ & $a_{14}$ & $a_{13}$ & $a_{13}$ & $a_{16}$ & $a_{15}$ & $a_{16}$ & $a_{15}$ & $a_{16}$ & $a_{15}$ & $a_{16}$ & $a_{15}$ \\
\hline $\mathrm{B} 3$ & $a_{2} \quad a_{2} \quad a_{0} \quad a_{1} \quad a_{2} \quad a_{2}$ & $a_{7}$ & $a_{7}$ & $a_{5}$ & $a_{6}$ & $a_{7}$ & $a_{7}$ & $a_{16}$ & $a_{15}$ & $a_{16}$ & $a_{15}$ & $a_{13}$ & $a_{13}$ & $a_{14}$ & $a_{14}$ & $a_{15}$ & $a_{16}$ & $a_{16}$ & $a_{15}$ \\
\hline B4 & $\begin{array}{llllll}a_{2} & a_{2} & a_{1} & a_{0} & a_{2} & a_{2}\end{array}$ & $a_{7}$ & $a_{7}$ & $a_{6}$ & $a_{5}$ & $a_{7}$ & $a_{7}$ & $a_{15}$ & $a_{16}$ & $a_{15}$ & $a_{16}$ & $a_{14}$ & $a_{14}$ & $a_{13}$ & $a_{13}$ & $a_{16}$ & $a_{15}$ & $a_{15}$ & $a_{16}$ \\
\hline u. & $a_{2} \quad a_{2} \quad a_{2} \quad a_{2} \quad a_{0} \quad a_{1}$ & $a_{7}$ & $a_{7}$ & $a_{7}$ & $a_{7}$ & $a_{5}$ & $a_{6}$ & $a_{16}$ & $a_{15}$ & $a_{15}$ & $a_{16}$ & $a_{15}$ & $a_{16}$ & $a_{16}$ & $a_{15}$ & $a_{13}$ & $a_{13}$ & $a_{14}$ & $a_{14}$ \\
\hline C6 & $a_{2} \quad a_{2} \quad a_{2} \quad a_{2} \quad a_{1} \quad a_{0}$ & $a_{7}$ & $a_{7}$ & $a_{7}$ & $a_{7}$ & $a_{6}$ & $a_{5}$ & 15 & $a_{16}$ & $a_{16}$ & $a_{15}$ & $a_{16}$ & $a_{15}$ & $a_{15}$ & $a_{16}$ & $a_{14}$ & $a_{14}$ & $a_{13}$ & $a_{13}$ \\
\hline & $a_{5}^{*} a_{6}^{*} a_{7}^{*} a_{7}^{*} a_{7}^{*} a_{7}^{*}$ & $a_{0}$ & us & $a_{4}$ & $a_{4}$ & 4 & $a_{4}$ & 17 & 117 & $a_{18}$ & $a_{18}$ & $a_{19}$ & $a_{20}$ & $A_{19}$ & $a_{20}$ & $a_{19}$ & $a_{20}$ & $a_{19}$ & $a_{20}$ \\
\hline $\mathrm{B} 2$ & $a_{6}^{*} a_{5}^{*} a_{7}^{*} a_{7}^{*} a_{7}^{*} a_{7}^{*}$ & $a_{3}$ & $a_{0}$ & $a_{4}$ & $a_{4}$ & $a_{4}$ & & 18 & 18 & $a_{17}$ & 17 & $a_{20}$ & $a_{19}$ & $a_{20}$ & $a_{19}$ & $a_{20}$ & $a_{19}$ & $a_{20}$ & $a_{19}$ \\
\hline & $a_{7^{*}} a_{7^{*}} a_{5}^{*} a_{6}^{*} a_{7^{*}} a_{7^{*}}$ & $a_{4}$ & $a_{4}$ & $a_{0}$ & $a_{3}$ & $a_{4}$ & $a_{4}$ & $a_{20}$ & $a_{19}$ & $a_{20}$ & $a_{19}$ & $a_{17}$ & $a_{17}$ & $a_{18}$ & $a_{18}$ & $a_{19}$ & $a_{20}$ & $a_{20}$ & $a_{19}$ \\
\hline D4 & $a_{7^{*}} a_{7^{*}} a_{6^{*}} a_{5} a_{7^{*}} a_{7^{*}}$ & $a_{4}$ & $a_{4}$ & $a_{3}$ & $a_{0}$ & $a_{4}$ & 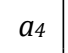 & 119 & $a_{20}$ & $a_{19}$ & $a_{20}$ & $a_{18}$ & $a_{18}$ & $a_{17}$ & $a_{17}$ & $a_{20}$ & $a_{19}$ & $a_{19}$ & $a_{20}$ \\
\hline & $a_{7^{*}} a 7^{*} a_{7^{*}} a_{7^{*}} a_{5^{*}} a_{6^{*}}$ & $a_{4}$ & $a_{4}$ & $a_{4}$ & $a_{4}$ & $a_{0}$ & 13 & 20 & $a_{19}$ & $a_{19}$ & $a_{20}$ & $a_{19}$ & $a_{20}$ & $a_{20}$ & $a_{19}$ & $a_{17}$ & $a_{17}$ & $a_{18}$ & $a_{18}$ \\
\hline Fo & $a_{7}^{*} a_{7}^{*} a_{7}^{*} a_{7}^{*} a_{6}^{*} a_{5}^{*}$ & & & $a_{4}$ & $a_{4}$ & & $a_{0}$ & $a_{19}$ & $a_{20}$ & $a_{20}$ & $a_{19}$ & $a_{20}$ & $a_{19}$ & $a_{19}$ & $a_{20}$ & $a_{18}$ & $a_{18}$ & $a_{17}$ & $a_{17}$ \\
\hline & $a_{13} * a_{14} * a_{16} * a_{15} * a_{16} * a_{15} *$ & $a_{17}$ & $a_{18} *$ & $a_{20} *$ & $a_{19}{ }^{*}$ & $a_{20} *$ & $a_{19} *$ & $a_{0}$ & $a_{8}$ & $a_{9}$ & $a_{9}$ & $a_{10}$ & $a_{11}$ & $a_{12}$ & $a_{10} *$ & $a_{10}$ & $a_{11}$ & $a_{12}$ & $10^{*}$ \\
\hline & $a_{13}{ }^{*} a_{14}{ }^{*} a_{15}{ }^{*} a_{16}{ }^{*} a_{15}{ }^{*} a_{16}{ }^{*}$ & $a_{17}$ & & & & & & $a_{8}$ & $a_{0}$ & $g_{0}$ & $g_{0}$ & 12 & $10^{*}$ & $a_{10}$ & $a_{11}$ & $a_{12}$ & $a_{10} *$ & $a_{10}$ & $a_{11}$ \\
\hline & $a_{14} * a_{13} * a_{16} * a_{15} * a_{15} * a_{16} *$ & $a_{18} *$ & & $a_{20}$ & $a_{19}$ & & & $a_{9}$ & $a_{9}$ & $a_{0}$ & $a_{8}$ & $a_{11}$ & $a_{10}$ & $a_{10} *$ & $a_{12}$ & $a_{10} *$ & $a_{12}$ & $a_{11}$ & $a_{10}$ \\
\hline & $a_{14} * a_{13} * a_{15} * a_{16} * a_{16} * a_{15} *$ & $a_{18} *$ & $a_{17} *$ & $a_{19} *$ & $a_{20}$ & $a_{20} *$ & $a_{19} *$ & $a_{9}$ & $a_{9}$ & $a_{8}$ & $a_{0}$ & $a_{10} *$ & $a_{12}$ & $a_{11}$ & $a_{10}$ & $a_{11}$ & $a_{10}$ & $a_{10}^{*}$ & $a_{12}$ \\
\hline & $a_{15}{ }^{*} a_{16}{ }^{*} a_{13}{ }^{*} a_{14}{ }^{*} a_{15}{ }^{*} a_{16}{ }^{*}$ & $a_{19} *$ & & $a_{17^{*}}$ & & & & $a_{10} *$ & $a_{12}$ & $a_{11}$ & $a_{10}$ & $a_{0}$ & $a_{0}$ & $a_{0}$ & $a_{9}$ & $a_{12}$ & $a_{10} *$ & $a_{11}$ & $a_{10}$ \\
\hline & $a_{16}{ }^{*} a_{15} * a_{13} * a_{14} * a_{16} * a_{15} *$ & $a_{20} *$ & & $a_{17}$ & & & & $a_{11}$ & $a_{10}$ & $a_{10}^{*}$ & $a_{12}$ & $a_{8}$ & $a_{0}$ & $a_{9}$ & $a_{9}$ & $a_{10}$ & $a_{11}$ & $a_{10} *$ & $a_{12}$ \\
\hline & $a_{15} * a_{16}^{*} a_{14} * a_{13} * a_{16} * a_{15} *$ & $a_{19} *$ & $a_{20}$ & $a_{18} *$ & $a_{17}$ & $a_{20} *$ & $a_{19} *$ & $a_{12}$ & $a_{10} *$ & $a_{10}$ & $a_{11}$ & $a_{9}$ & $a_{9}$ & $a_{0}$ & $a_{8}$ & $a_{11}$ & $a_{10}$ & $a_{12}$ & $a_{10} *$ \\
\hline & $a_{16} * a_{15} * a_{14} * a_{13} * a_{15} * a_{16} *$ & $a_{20} *$ & & $a_{18}$ & $a_{17}$ & & $a_{20} *$ & $a_{10}$ & $a_{11}$ & $a_{12}$ & $a_{10}^{*}$ & $a_{9}$ & $a_{9}$ & $a_{8}$ & $a_{0}$ & $a_{10} *$ & $a_{12}$ & $a_{10}$ & $a_{11}$ \\
\hline D & $a_{15} * a_{16} * a_{15} * a_{16} * a_{13} * a_{14} *$ & $a_{19} *$ & $a_{20} *$ & $a_{19} *$ & $a_{20} *$ & $a_{17}$ & $a_{18} *$ & $a_{10} *$ & $a_{12}$ & $a_{10}$ & $a_{11}$ & $a_{12}$ & $a_{10} *$ & $a_{11}$ & $a_{10}$ & $a_{0}$ & $a_{8}$ & $a_{9}$ & $a_{9}$ \\
\hline Le & $a_{16} * a_{15} * a_{16} * a_{15} * a_{13} * a_{14} *$ & $a_{20}$ & $a_{19}$ & $a_{20}$ & $a_{19} *$ & $a_{1}$ & $a_{18} *$ & $a_{11}$ & $a_{10}$ & $a_{12}$ & $a_{10} *$ & $a_{10}$ & $a_{11}$ & $a_{10} *$ & $a_{12}$ & $a_{8}$ & $a_{0}$ & $a_{9}$ & $a_{9}$ \\
\hline 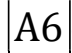 & $a_{15} * a_{16} * a_{16} * a_{15} * a_{14} * a$ & $a_{19}$ & u & $a_{20}$ & k & $a_{1}$ & $a_{17^{*}}$ & $a_{12}$ & $a_{10}^{*}$ & $a_{11}$ & $a_{10}$ & $a_{11}$ & $a_{10}$ & $a_{12}$ & $a_{10}^{*}$ & $a_{9}$ & $a_{9}$ & $a_{0}$ & $a_{8}$ \\
\hline & $a_{16} * a_{15} * a_{15} * a_{16} * a_{14} * a_{13} *$ & $a_{20} *$ & & $a_{19} *$ & & $a_{18}^{*}$ & $a_{17^{*}}$ & $a_{10}$ & $a_{11}$ & $a_{10} *$ & $a_{12}$ & $a_{10} *$ & $a_{12}$ & $a_{10}$ & $a_{11}$ & $a_{9}$ & $a_{9}$ & $a_{8}$ & $a_{0}$ \\
\hline
\end{tabular}




\section{Supplementary Section 2}

\section{Elastic constants and Scattergood and Bacon's average moduli}

The $\mathrm{UO}_{2}$ elasticity is defined considering $C_{11}, C_{12}$ et $C_{44}$ elastic constants using Voigt convention in orthotropic cubic material. Elastic constants as a function of temperature are provided in ref. [1].

Table S2.1: Elastic constants at $1373 \mathrm{~K}, 1600 \mathrm{~K}$ and $1973 \mathrm{~K}$ from [S1].

\begin{tabular}{cccc}
\hline Elastic constants (GPa) & $\mathbf{1 3 7 3 ~ K}$ & $\mathbf{1 6 0 0 ~ K}$ & $\mathbf{1 9 7 3 ~ K}$ \\
\hline $\boldsymbol{C}_{\mathbf{1 1}}$ & 297 & 278 & 248 \\
$\boldsymbol{C}_{\boldsymbol{1 2}}$ & 107 & 104 & 98 \\
$\boldsymbol{C}_{\boldsymbol{4 4}}$ & 56 & 54 & 50 \\
\hline
\end{tabular}

Table S2.2: BS elastic constants for the three slip modes at $1973 \mathrm{~K}$ [S2].

\begin{tabular}{|c|c|c|c|c|c|c|}
\hline & & & & $1 / 2<110>\{100\}$ & $1 / 2<110>\{110\}$ & $1 / 2<110>\{111\}$ \\
\hline $1373 \mathrm{~K}$ & & 72.7 & & 0.25 & 0.32 & 0.29 \\
\hline $1600 \mathrm{~K}$ & $\mu^{B S}(\mathrm{GPa})$ & 68.5 & $v^{\mathrm{BS}}$ & 0.26 & 0.32 & 0.29 \\
\hline $1973 \mathrm{~K}$ & & 61.4 & & 0.27 & 0.32 & 0.30 \\
\hline
\end{tabular}

References

[S1] M.T. Hutchings, High-temperature studies of UO2 and ThO2 using neutron scattering techniques, J. Chem. Soc. Faraday Trans. 2 Mol. Chem. Phys. 83 (1987) 1083-1103. https://doi.org/10.1039/F29878301083.

[S2] D.J. Bacon, D.M. Barnett, R.O. Scattergood, Anisotropic continuum theory of lattice defects, Prog. Mater. Sci. 23 (1980) 51-262. https://doi.org/10.1016/00796425(80)90007-9. 


\section{Supplementary Section 3}

\section{DD simulations}

\subsection{Effect of the damping coefficient used in the dislocation mobility law}

Thanks to the Orowan law equation $\left(\dot{\gamma}=\rho_{m} b v\right)$ and the viscous dislocation mobility law $(v=\tau b / B)$ we can estimate the stress due to the mobility (equation (1)).

$$
\tau_{v}=\frac{\dot{\gamma} B}{\rho_{m} b^{2}}=\frac{\left(\frac{4.5}{0.5}\right) 1.510^{-5}}{10^{11}\left(\frac{5.4710^{-10}}{\sqrt{2}}\right)^{2}}=9.010^{3} \mathrm{~Pa} \approx 10^{-2} \mathrm{MPa}
$$

The stress derived from the Taylor equation with an Hirth coefficient equal to 0.1 is given in equation (2).

$$
\tau_{f}=\alpha \mu b \sqrt{\rho_{f}}=0.172 .710^{9}\left(\frac{5.4710^{-10}}{\sqrt{2}}\right) \sqrt{10^{12}}=2.7 \mathrm{MPa}
$$

A ratio $\frac{\tau_{v}}{\tau_{f}} \approx 310^{-3}$ is small enough in order to have some results independent from the damping coefficient.

\subsection{Volume geometry}

Table S3: Simulated volume geometry

\begin{tabular}{cccc}
\hline Mobile system & $1 / 2[011](100)$ & $1 / 2[011](0 \overline{1} 1)$ & $1 / 2[\overline{1} 01](111)$ \\
\hline After rotation & {$[\overline{4} 115](\overline{1} \overline{4} 8)$} & {$[\overline{4} 115](1233)$} & {$[\overline{6} 06](666)$} \\
Simulated volume & $L_{x}=10,5 \mu \mathrm{m}$ & $L_{x}=10 \mu \mathrm{m}$ & $L_{x}=10 \mu \mathrm{m}$ \\
geometry & $L_{y}=10 \mu \mathrm{m}$ & $L_{y}=11,38 \mu \mathrm{m}$ & $L_{y}=11,06 \mu \mathrm{m}$ \\
& $L_{z}=11,82 \mu \mathrm{m}$ & $L_{z}=12,32 \mu \mathrm{m}$ & $L_{z}=613,29 \mu \mathrm{m}$ \\
\hline
\end{tabular}




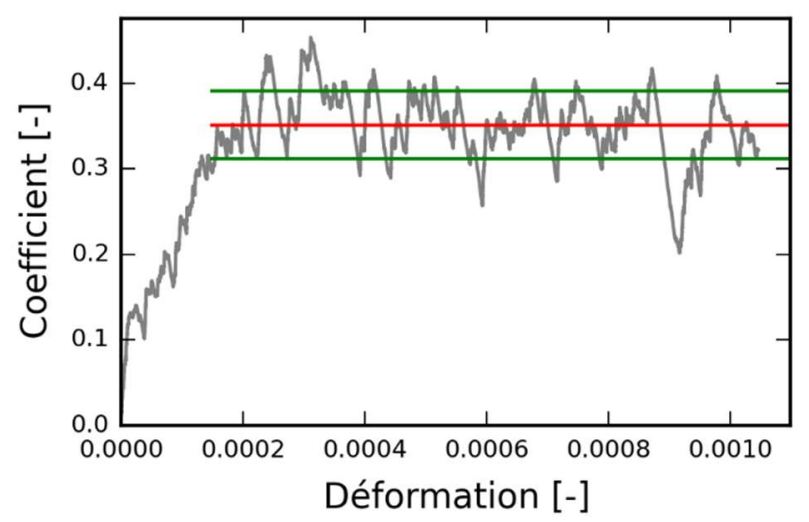

Figure S3: Methods for estimating coefficient values (here $\alpha_{2}$ at $1373 \mathrm{~K}$ ) from stress strain curves. Stress fluctuations (in grey) are attributed to dislocation avalanches. The mean value is in red and the standard deviation is emphasized in green. 


\section{Supplementary Section 4}

\section{Effect of temperature on strengthening coefficients}

Table S4.1: Coefficient values for $1373 \mathrm{~K}$ and $1600 \mathrm{~K} . \beta_{1}$ and $\beta_{2}$ represent the character of the mobile dislocations and the forest dislocations respectively $\left(0^{\circ} \rightarrow\right.$ screw $-90^{\circ} \rightarrow$ edge). $\beta_{12}$ is the angle between the slip planes. The recombination rate is provided at $1373 \mathrm{~K}$, and the junction density at $1973 \mathrm{~K}$ with $0.1 \%$ of deformation.

\begin{tabular}{|c|c|c|c|c|c|c|c|c|c|}
\hline & & $\alpha(373 K)$ & $\alpha(1600 \mathrm{~K})$ & $\beta_{1}$ & $\beta_{2}$ & $\beta_{12}$ & $\beta_{j}$ & $\begin{array}{c}\text { recomb. } \\
\text { rate }\end{array}$ & $\begin{array}{c}\rho_{\text {junction }} \\
\left(m^{-2}\right)\end{array}$ \\
\hline$\alpha_{2}$ & glissile $_{I / I}^{I I}$ & $0.35 \pm 0.04$ & $0.35 \pm 0.06$ & 45 & 45 & 90 & 90 & 42.2 & $5.5 \times 10^{11}$ \\
\hline$\alpha_{3}$ & $\operatorname{Hirth}_{I I / I I}$ & $0.14 \pm 0.03$ & $0.15 \pm 0.02$ & 90 & 90 & 90 & 90 & 0.5 & $1.0 \times 10^{10}$ \\
\hline$\alpha_{4}$ & sessile ${ }_{I I / I I}^{\{112\}}$ & $0.26 \pm 0.03$ & $0.26 \pm 0.04$ & 35 & 35 & 60 & 90 & 34.5 & $4.0 \times 10^{11}$ \\
\hline$\alpha_{5}$ & \multirow{2}{*}{ collinear $r_{I / I I}^{90^{\circ}}$} & $0.84 \pm 0.05$ & $0.91 \pm 0.05$ & 0 & 0 & 90 & - & 73.8 & 0.0 \\
\hline$\alpha_{5}^{*}$ & & $0.86 \pm 0.06$ & $0.86 \pm 0.06$ & 0 & 0 & 90 & - & 73.8 & 0.0 \\
\hline$\alpha_{6}$ & \multirow{2}{*}{$\operatorname{Hirth}_{I / I I}$} & $0.11 \pm 0.01$ & $0.09 \pm 0.02$ & 90 & 0 & 90 & 45 & 6.7 & $2.0 \times 10^{9}$ \\
\hline$\alpha_{6} *$ & & $0.14 \pm 0.02$ & $0.14 \pm 0.03$ & 0 & 90 & 90 & 45 & 6.7 & $1.5 \times 10^{10}$ \\
\hline$\alpha_{7}$ & \multirow{2}{*}{ glissile $_{I / I I}^{I}$} & $0.38 \pm 0.05$ & $0.40 \pm 0.04$ & 45 & 90 & 45 & 45 & 47.3 & $6.0 \times 10^{11}$ \\
\hline$\alpha_{7}^{*}$ & & $0.39 \pm 0.05$ & $0.40 \pm 0.05$ & 90 & 45 & 45 & 45 & 47.3 & $6.0 \times 10^{11}$ \\
\hline$\alpha_{8}$ & collinear ${ }_{I I I / I I I}^{70^{\circ}}$ & $0.78 \pm 0.05$ & $0.78 \pm 0.05$ & 0 & 0 & 70 & - & 77.0 & 0.0 \\
\hline$\alpha_{9}$ & $\operatorname{Hirth}_{I I I / I I I}$ & $0.16 \pm 0.02$ & $0.15 \pm 0.02$ & 60 & 60 & 70 & 45 & 7.8 & $8.0 \times 10^{10}$ \\
\hline$\alpha_{10}$ & \multirow{2}{*}{ glissile $e_{I I I / I I I}^{I}$} & $0.29 \pm 0.03$ & $0.30 \pm 0.04$ & 60 & 0 & 70 & 60 & 38.7 & $4.5 \times 10^{11}$ \\
\hline$\alpha_{10} *$ & & $0.32 \pm 0.04$ & $0.31 \pm 0.03$ & 0 & 60 & 70 & 60 & 38.7 & $5.0 \times 10^{11}$ \\
\hline$\alpha_{12}$ & glissile $_{I I I / I I I}^{I, L}$ & $0.35 \pm 0.05$ & $0.38 \pm 0.04$ & 60 & 60 & 70 & 90 & 41.4 & $5.5 \times 10^{11}$ \\
\hline$\alpha_{13}$ & \multirow{2}{*}{ collinear ${ }_{I / I I I}^{55^{\circ}}$} & $0.78 \pm 0.07$ & $0.79 \pm 0.05$ & 0 & 0 & 55 & - & 79.6 & 0.0 \\
\hline$\alpha_{13} *$ & & $0.75 \pm 0.05$ & $0.75 \pm 0.05$ & 0 & 0 & 55 & - & 79.6 & 0.0 \\
\hline$\alpha_{14}$ & \multirow{2}{*}{$\operatorname{Hirth}_{I / I I I}$} & $0.09_{ \pm 0.01}$ & $0.10_{ \pm 0.02}$ & 90 & 0 & 55 & 45 & 4.3 & $3.0 \times 10^{9}$ \\
\hline$\alpha_{14} *$ & & $0.12 \pm 0.01$ & $0.12 \pm 0.01$ & 0 & 90 & 55 & 45 & 4.3 & $1.1 \times 10^{10}$ \\
\hline$\alpha_{15}$ & \multirow{2}{*}{ glissile $I / I I I I a$} & $0.39 \pm 0.05$ & $0.40 \pm 0.05$ & 90 & 60 & 55 & 60 & 44.2 & $6.0 \times 10^{11}$ \\
\hline$\alpha_{15} *$ & & $0.36 \pm 0.05$ & $0.36 \pm 0.05$ & 60 & 90 & 55 & 60 & 44.2 & $5.0 \times 10^{11}$ \\
\hline$\alpha_{16}$ & \multirow{2}{*}{ glissile ${ }_{I / I I I b}^{I I I}$} & $0.33 \pm 0.04$ & $0.32 \pm 0.04$ & 0 & 60 & 55 & 60 & 39.5 & $5.0 \times 10^{11}$ \\
\hline$\alpha_{16} *$ & & $0.28 \pm 0.04$ & $0.27 \pm 0.03$ & 60 & 0 & 55 & 60 & 39.5 & $4.5 \times 10^{11}$ \\
\hline$\alpha_{17}$ & \multirow{2}{*}{ collinear $r_{I I / I I I}^{35^{\circ}}$} & $0.63 \pm 0.05$ & $0.69 \pm 0.05$ & 0 & 0 & 35 & - & 80.8 & 0.0 \\
\hline$\alpha_{17} *$ & & $0.65 \pm 0.06$ & $0.65 \pm 0.06$ & 0 & 0 & 35 & - & 80.8 & 0.0 \\
\hline$\alpha_{18}$ & \multirow{2}{*}{$\operatorname{Hirth}_{I I / I I I}^{\{012\}}$} & $0.17 \pm 0.02$ & $0.17 \pm 0.02$ & 55 & 90 & 90 & 66 & 0.16 & $1.1 \times 10^{10}$ \\
\hline$\alpha_{18} *$ & & $0.14 \pm 0.01$ & $0.15 \pm 0.01$ & 90 & 55 & 90 & 66 & 0.16 & $5.0 \times 10^{9}$ \\
\hline$\alpha_{19}$ & \multirow{2}{*}{ sessile $_{I I / I I I}^{\{113\}}$} & $0.32 \pm 0.05$ & $0.32 \pm 0.04$ & 55 & 30 & 90 & 73 & 37.4 & $5.0 \times 10^{11}$ \\
\hline$\alpha_{19} *$ & & $0.33 \pm 0.05$ & $0.32 \pm 0.03$ & 30 & 55 & 90 & 73 & 37.4 & $5.0 \times 10^{11}$ \\
\hline$\alpha_{20}$ & \multirow{2}{*}{ glissile $I I I / I I I$} & $0.30 \pm 0.06$ & $0.30 \pm 0.04$ & 0 & 60 & 35 & 60 & 40.3 & $4.0 \times 10^{11}$ \\
\hline$\alpha_{20} *$ & & $0.25 \pm 0.03$ & $0.28 \pm 0.04$ & 60 & 0 & 35 & 60 & 40.3 & $4.0 \times 10^{11}$ \\
\hline
\end{tabular}


Table S4.2: Mean values of coefficients at 1973K, recombination rate, cross state (CS) repulsion (R) and CS and R together for each king of interaction at $1373 \mathrm{~K}$.

\begin{tabular}{lccccc}
\hline & $\bar{\alpha}(1973 \mathrm{~K})$ & $\begin{array}{c}\text { Recombination } \\
\text { rate }\end{array}$ & $\begin{array}{c}\text { Cross } \\
\text { states }\end{array}$ & Repulsion & Recomb+CS \\
\hline Colinear & 0.75 & 77.8 & 3.4 & 20.8 & 81.2 \\
Glissiles & 0.35 & 41.9 & 31.3 & 26.7 & 73.2 \\
Sessiles & 0.29 & 35.9 & 36.7 & 27.4 & 72.6 \\
Sessiles and glissiles & 0.33 & 40.6 & 32.5 & 26.9 & 73.1 \\
Hirth & 0.13 & 3.9 & 54.9 & 28.6 & 58.8 \\
\hline
\end{tabular}

\section{Supplementary Section 5}

\section{Hirth junction interaction maps}

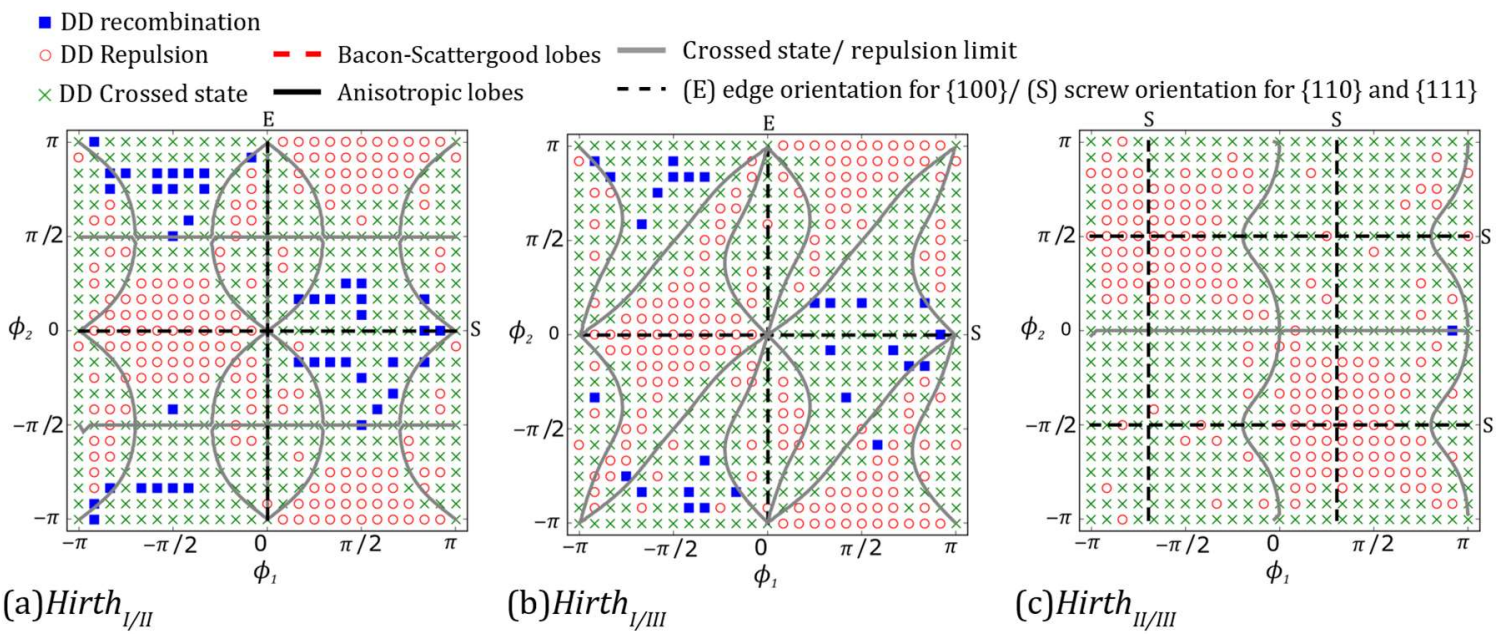

Figure S5: $\mathrm{UO}_{2}$ additional Hirth maps without recombination lobes. 


\section{Supplementary Section 6}

\section{Influence of moduli and crystallography on the coefficient value}

Figure S6.1 shows the influence of $v^{B S}$ and $\beta_{1}$ on all $\mathrm{UO}_{2}$ coefficient values. No clear global tendency can be seen due to these parameters.
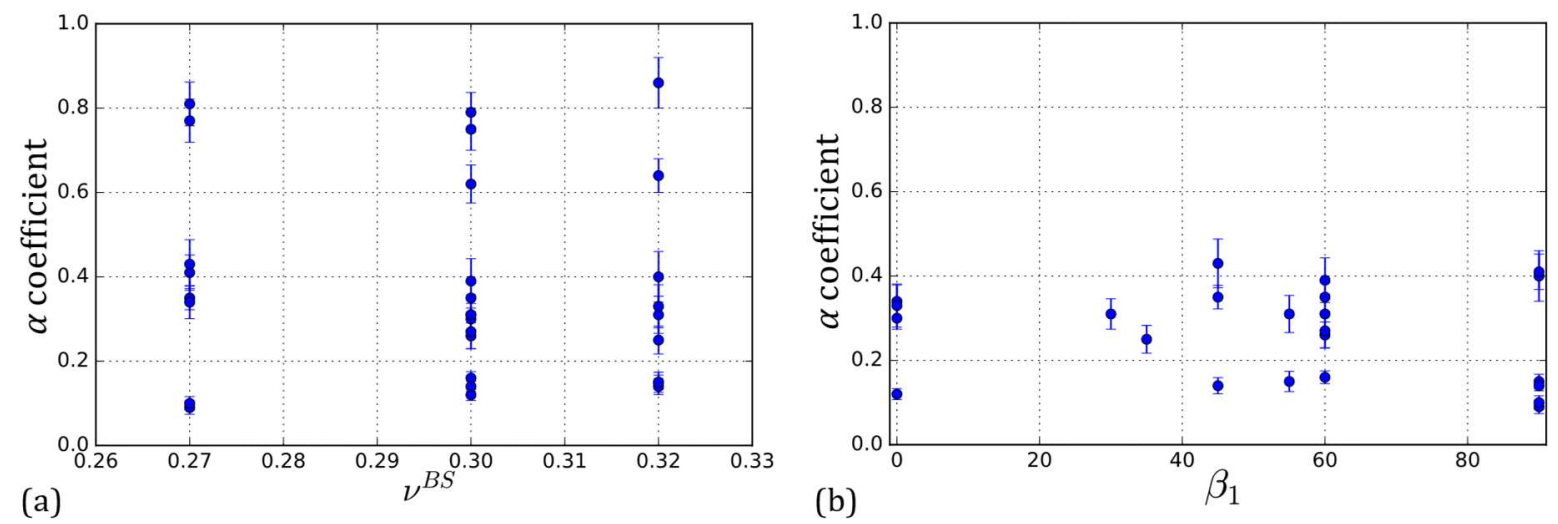

Figure S6.1: All coefficient values versus (a) the Poisson's ratio $\left(v^{B S}\right)$ and $(b)$ the character of the mobile dislocations $\left(\beta_{1}\right)$.

Hirth junction deserve a specific analysis because those coefficients are particular since they do not form a lot of junction with the exception of the Hirth character of the mobile dislocations is clear and induces the asymmetry, the strong effect for a6/a6* coefficient is also related with the very different number of junctions formed (see text).

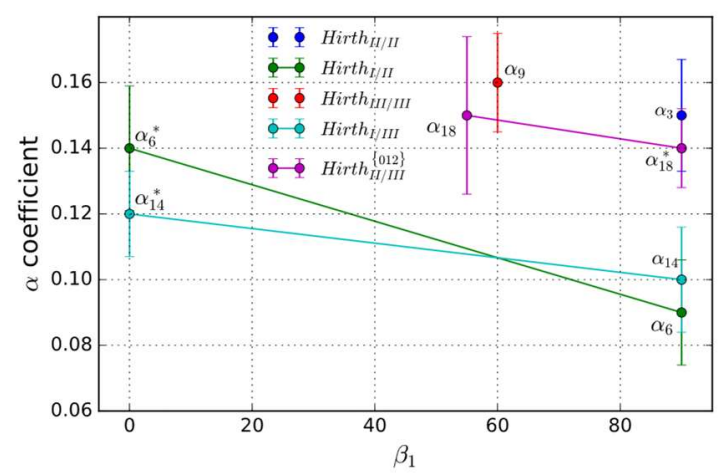

Figure S6.2: Hirth coefficient values versus the character of the mobile dislocations $\left(\beta_{1}\right)$. 